See discussions, stats, and author profiles for this publication at: https://www.researchgate.net/publication/276074930

\title{
Game Theory for Energy Efficiency in Wireless Sensor Networks: Latest
} Trends

Article in Journal of Network and Computer Applications · May 2015 DOI: 10.1016/j.jnca.2015.03.011

\section{CITATIONS}

85

3 authors:

Tarek Alskaif

Utrecht University

30 PUBLICATIONS 294 CITATIONS

SEE PROFILE

Boris Bellalta

University Pompeu Fabra

184 PUBLICATIONS 2,145 CITATIONS

SEE PROFILE

\section{READS}

1,184

Some of the authors of this publication are also working on these related projects:

Project PARticipatory platform for sustainable ENergy managemenT (PARENT) View project

Project Smart Solar Charging View project 


\title{
Game Theory for Energy Efficiency in Wireless Sensor Networks: Latest Trends
}

\author{
Tarek AlSkaifa, ${ }^{\mathrm{a}, *}$, Manel Guerrero Zapata ${ }^{\mathrm{a}}$, Boris Bellalta ${ }^{\mathrm{b}}$ \\ ${ }^{a}$ Department of Computer Architecture \\ Polytechnic University of Catalonia (UPC, BarcelonaTech) \\ 08034 Barcelona, Spain \\ ${ }^{b}$ Universitat Pompeu Fabra (UPF) \\ 08018 Barcelona, Spain
}

\begin{abstract}
In the area of Wireless Sensor Networks (WSNs, improving energy efficiency and network lifetime is one of the most important and challenging issues. Most of the considered WSNs are formed by nodes with limited resources, in which each node plays dual rule of both sensing the environment and relaying traffic to the sink from other nodes. On the one hand, the nodes need to stay alive as long as possible by achieving energy efficiency. On the other hand, they have to provide the required service. This conflict of interest makes game theory very useful in WSNs. Moreover, the nodes usually have to make decisions with very limited information about the state of the network. Game theory has been used recently in a remarkable amount of research in this area. In this survey, we review the most recent papers about using game theory in WSNs to achieve a trade-off between maximizing the network lifetime and providing the required service. The paper contains a complete taxonomy of games applied to this specific research problem. It summarizes and compares the different published proposals along with tables and statistical charts showing in which domains game theory has been applied recently. Overall, the paper will give researchers a clear view of the newest trends in this research area, underlining its main challenges and hopefully fostering discussions and new research directions.
\end{abstract}

Keywords: Wireless sensor networks; Game theory; Energy efficiency; Network lifetime

\section{Introduction}

Wireless Sensor Networks (WSNs) have met a growing interest in the last decade due to their applicability to a large class of contexts, such as environment monitoring, object tracking, traffic control, and health applications, among others. Since it is usually difficult and costly to replace faulty sensors once they are deployed, reducing the energy consumption in WSNs is of paramount importance in order to maximize the 10 network lifetime [1]. The lifetime of a sensor network is defined as the time until any or a given number of sensors in the network dies.

This research area has drawn a lot of attention in the last few years with many researchers developing solu15 tions to extend nodes' battery life as much as possible. A survey that offers a comprehensive view of energy- 35 saving solutions in WSNs while taking applications' requirements into consideration is presented in [2]. It presents a novel classification of energy-conservation

\footnotetext{
Corresponding author. Tel.: +34 603640839 .

Email addresses: tarek@ac.upc.edu (Tarek AlSkaif ), guerrero@ac. upc . edu (Manel Guerrero Zapata), boris.bellalta@upf .edu (Boris Bellalta)
} of energy-saving mechanisms is summarized as follows: radio optimization, data reduction, sleep/wakeup schemes, energy-efficient routing and energy repletion. So far, different approaches and mathematical methods have been used to characterize this problem, ranging from computational intelligence and optimization methods to game theory. However, the main challenge is represented in the trade-off between energy conservation and Quality of Service (QoS) which makes the problem of energy conservation more complicated.

Game theory [3], which has been mainly studied and applied in economics, politics and sociology, has recently emerged as an useful tool in analyzing modern wireless networks since it provides analytical tools to model interactions among entities with conflicting interests that compete for the limited network resources (i.e., energy and/or bandwidth). Like resourceconstrained nodes in a wireless network that might -for instance- decide not to forward packets in order to pre(4]. In some cases, nodes may seek to optimize the overall network performance. In other cases, nodes may behave maliciously, seeking to ruin network performance for other users [5]. Game

May 3, 2015 
theory offers a wide range of formulations and models well as network-wide performance in a flexible manner [6]. In addition, game theory allows us to model scenarios in which there is no centralized entity with a full picture of network conditions [5].

There are some previously published surveys in the literature about using game theory in WSNs [6, 7] and in ad hoc networks [8]. However, the main difference between these surveys and this one is that none of the previous surveys has explicitly considered or identified the energy efficiency and lifetime maximization problems in WSNs. For example, [7] surveys the use of game theory in WSNs in general, without focusing on energy efficiency. [6] surveys the amount of work done using game theory in WSNs before 2008, focusing on two main domains energy efficiency and security. However, there are a considerable amount of research and valuable developments in this area in the last few years that non of these surveys covers. In addition, in this survey we cover proposals that use various game theory concepts and models which are not mentioned in previous surveys like Bargaining games, Bayesian Nash Equilibrium, the core, and correlated equilibrium, among others.

This paper provides a survey of recent research stud-100 ies employing game theory to improve energy conservation and prolong the network lifetime in WSNs. The paper first presents a brief overview about WSNs in Section 2. Then, a categorization for game theory models with non-cooperative, cooperative, and cooper-105 ation enforcement games as the top main categories, is presented in Section 3. Later, the literature is surveyed at four levels: i) Power Control and Medium Access Control (MAC) in Section 4, ii) Routing and Clustering in Section 5, iii) Coverage and Topology Control 110 in Section 6, and iv) Data Aggregation, Security, Task Allocation and Energy Harvesting in Section 7. Each level is further divided into three parts based on the applications of non-cooperative, cooperative and cooperation enforcement games. In each level the papers 115 of game, game solution strategies and energy savings methods used. Besides, the articles in the tables are ordered according to the year of publication. The survey is completed with the conclusions in Section 8. The 120

90 paper will give to the reader a brief but comprehensive view of the state of the art in all aspects of this research area, and shed the light on its main current challenges and future trends. We also hope it will foster discussion and new research ideas among researchers, pro-125 viding them with a clear description of the state of the art. Fig 1 summarizes the organization and the structure of the survey.

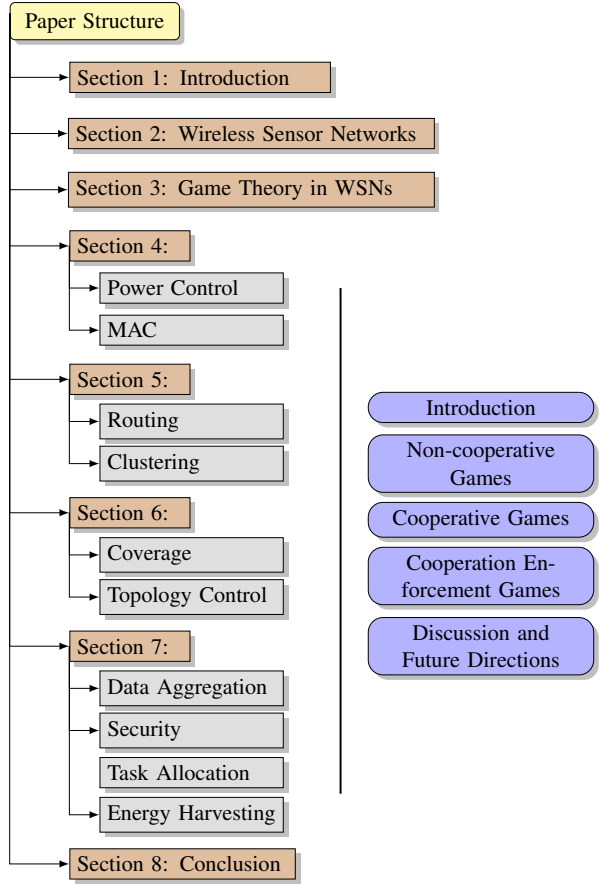

Figure 1: The organization of the paper

\section{Wireless Sensor Networks (WSNs)}

A Wireless Sensor Network (WSN) is a wireless network where all or most of its nodes have sensors (ranging from scalar sensors that can sense temperature or relative humidity to video cameras). Sensor network is typically formed by a large number of small in size, low-cost, battery-powered and resourceconstrained nodes that might be randomly disposed or strategically placed all over the region of interest in order to perform certain tasks like monitoring, detecting events, generating reports and communicating, likely via multi-hop wireless links, with one or more destination nodes -that will collect the sensed information and possibly perform specific requests for certain sensed data- called sinks.

In some scenarios, when multiple surrounding nodes detect the same event, one of them generates a final report after collaborating with the other nodes. The sink can process the report and possibly send it out through either high-quality wireless or wired links to external centers for further processing. Nodes are static most of the time, whereas mobile nodes can be deployed according to application requirements. A sink can also be either static or mobile, and one or several sinks could be deployed together within the same network.

The topology of sensor networks could be classified as a: (i) flat topology, where nodes are all of the same level and behave according to same rules (i.e., generating and forwarding data), (ii) cluster-based topology, where there are different categories of sensor nodes (SNs), and cluster heads (CHs). SNs communicate 
mainly or exclusively to its $\mathrm{CH}$-usually the $\mathrm{CH}$ closest to itself. That divides the network region in clusters and the $\mathrm{CHs}$ perform all inter-cluster communications and might aggregate its cluster data before sending it ${ }_{185}$ to other $\mathrm{CHs}$ or to the sink.

Typically, a flat topology is used in homogeneous networks, where all nodes have the same capabilities. Cluster topologies are used in heterogeneous networks, as cluster heads have more capabilities in terms of en-190 ergy, communication and processing power. The use of these higher-capacity nodes can greatly increase the network reliability and lifetime but imposes additional cost and challenges like deciding how many and how they should be deployed, and in most cases require specific routing protocols. The reader can refer to [1, 9, 10] for further reading about WSNs.

\section{Game theory in Wireless Sensor Networks}

Game theory has been applied intensively in wire- ${ }_{200}$ less networks in the last decade. Previous work done in this area shows that it enables an intelligent behavior in challenging environments. The book entitled "Game Theory for Wireless Engineers" [5], presents game theoretic models and their application to modern wireless ${ }_{205}$ networks. A survey that demonstrates how game theory could be effectively applied in wireless networks is provided in [11]. It explains what are the best fields under the different OSI layers for applying game theory.

Game theory is considered a preferable approach for WSNs -in comparison with other types of wireless networks- for the following reasons. Firstly, solutions designed for WSNs should be fully or partially distributed. Secondly, nodes in WSNs are typ- ${ }_{215}$ ically resource-constrained, fixed, and homogeneous in terms of battery life and other hardware resources. Hence, nodes have conflicting interests between conserving energy in order to maximize the lifetime of the network, and between providing the required $\mathrm{QOS}_{220}$ However, researchers still have some challenges in selecting the appropriate game model and defining the utility function for each specific problem. This is because these models need to guarantee that the system converges to a desirable solution within reasonable ${ }_{225}$ time.

The following subsections will provide a brief overview about the various classifications and the different concepts of game theory which are illustrated in Fig. 2 .

\subsection{Main Classification of Games}

In general, games have been classified in different ways in the literature. The main classification of game theory divides the games into two main classes Noncooperative games and Cooperative games. In this survey we add a new class to that classification: Cooperation Enforcement games. We found that there is a significant number of articles that fall under this class, thus we hope that it will provide to the reader with a good understanding of game theory models. In the following subsections we describe the different concepts in each class with examples. Table 1 will guide the readers through the proposals that use these concepts in WSNs.

\subsubsection{Non-cooperative Games}

This class of games focuses on each player's individual utility rather than the utility of the whole network. In non-cooperative games individual players may act selfishly (i.e., deviate alone from a proposed solution if it is in their own interest, and do not coordinate their actions with the other players). In this context, nodes in an energy constraint multi-hop wireless network will probably act selfishly for the following reason: from a node's point of view, forwarding the incoming packets affects its own resources. Therefore, it may not be of the node's interest to forward all the incoming packets. In contrast, if it rejects forwarding other's packets, it will negatively affect network connectivity. This dilemma is called the Forwarder's Dilemma [4]. Nevertheless, when nodes belong to different authorities or clusters, it may be preferable to look for their interest. The technical report presented in [4] carefully explains how situations of this kind can be modeled by making use of game theory.

In general, a game consists of two components: i) participants called players, in our scenario a set of a wireless nodes $\mathcal{N}=\left\{n_{1}, \ldots, n_{N}\right\}$, where $N=|\mathcal{N}|$ is the number of nodes participating in the game, ii) a set of available actions for each player called strategies $\mathcal{S}=\left\{s_{1}, \ldots, s_{S}\right\}$ (e.g., the decision to forward packets or not), and iii) an associated amount of benefit or gain which each player receives at the end of the game, called payoff or utility $\mathcal{U}=\left\{u_{1}, \ldots, u_{U}\right\}$, which is a function that measures the degree of satisfaction for each available strategy in terms of the considered performance metrics (e.g., energy, delay, target signalto-noise ratio). Each player try to choose his best available action (i.e., the one which give a player the highest payoff, called best response). The best action for any given player depends, in general, on the other players' actions. So when choosing an action a player must have in mind the actions the other players will choose.

Nash equilibrium (NE) is a central solution concept in game theory. It captures the notion of a stable solution, from which no single player can individually improve his welfare by deviating [3]. Formally, a strategy vector $s \in \mathcal{S}$ is said to be a Nash Equilibrium if for any player $i$, and for each of its strategies $s_{i}^{\prime} \in \mathcal{S}$, we have that:

$$
u_{i}\left(s_{i}, s_{-i}\right) \geq u_{i}\left(s_{i}^{\prime}, s_{-i}\right)
$$


Nash equilibrium represents a certain stable operating ${ }_{285}$ necessarily the best operating point from a social perspective and for the WSNs, but it is at least the one which all players agree on. Nash theorem says that every finite game in strategic form has a Nash equi-290 librium in either mixed or pure strategies [12]. In the previous example (the Forwarder's Dilemma game), the Nash equilibrium point is when each node (player) drops other nodes' packets. A game has a Nash equilibrium in a pure strategy, when each player determin-295 istically plays his chosen strategy. When players are allowed to randomize and each player picks a certain probability distribution over his set of strategies, such a choice is called mixed strategy.

One method for identifying the desired Nash equi-зо0 librium point in a game, and ensuring that the solution maximizes the utilities for both players is to compare strategy profiles using the concept of Pareto-optimality [4]. In a Pareto-optimal strategy profile, the payoff of a given player can not be increased without decreas-305 ing the payoff of at least one other player. A game can have several Pareto-optimal strategy profiles, and it is important to note that a Pareto-optimal strategy profile is not necessarily a Nash equilibrium. In the Forwarder's Dilemma example, the strategy profile when ${ }_{310}$ both nodes forward packets of each other is Paretooptimal, but not a Nash equilibrium.

Potential games [3, 13] are games that admit a potential function, which in turn can be used to prove that the best-response dynamics converge to an equilibrium ${ }_{315}$ point. Potential games pose many interesting properties. For example, a pure Nash equilibrium always exists. Potential games can be classified into Exact Potential Game (EPG), when a given player switches from an action to another, the change in the potential ${ }_{320}$ 265 function equals the change in the utility of that player, and Ordinal Potential Game (OPG), when the changes in the potential function and the changes in the utility of that player are positive.

\subsubsection{Cooperative Games}

270 In contrast, cooperative games consider the utility of all players with the goal of maximizing the entire network's pay-off while maintaining fairness. The objective of cooperative game theory is to study ways to sustain cooperation among agents willing to cooperate. 330

275 The main question is how the benefits and the costs of a joint effort can be divided among participants, taking into account individual and group incentives, as well as various fairness properties [3].

Cooperative game theory is also known as coali-335 280 tional game theory. A coalitional game theory is designed to model situations in which players form groups (i.e., coalitions) rather than acting individually. A central notion in coalitional game theory is the notion of the core. The core is the set of payoff alloca- 340 tions that guarantees that no group of players has an incentive to leave its coalition to form another coalition. Therefore, if we can find a core in a coalitional game, no coalition will break away, and it will choose the action that all of its members prefer. In addition, by repeating the coalitional game, a certain "stable" state is achieved, where no player can improve its utility in the following repetitions [5]. However, the core solution can suffer from some drawbacks, like having an unfair allocation and being difficult to achieve, among others.

The other solution concept for coalitional game theory is Shapley value [14], which is one of the efficient solutions that are used in many proposals included in this survey. However, the complexity of calculating the Shapley value increases with the number of players, therefore it is recommended only for cases where the number of players is low.

Another widely applicable concept of cooperative games is bargaining games. The bargaining problem studies a situation where two or more players need to select one of the many possible outcomes of a joint collaboration. For example, players try to come to an agreement on a fair resource sharing inside a cluster. If the individuals reach an agreement, both of them can gain a higher benefit than playing the game without cooperation. The solution of this type of games is called Nash Bargaining Solution (NBS) [3, 15], in which no action taken by one of the individuals is imposed without the consent of the other.

The main difficulty of cooperative games is that nodes require to perform some extra computations and agreements between each other. However, the reader will find through the survey that when cooperative games are used, they usually give to the nodes a fair utility, allowing a noticeable improvement in energy conservation.

\subsubsection{Cooperation Enforcement Games}

This class considers players that would normally behave selfishly but they are enforced to cooperate, while still striving to maximize their outcomes from the game. Cooperation enforcement mechanisms are also designed to encourage greater cooperation by individuals. As we previously mentioned, in multi-hop wireless networks, each node serves as a source/destination for traffic as well as a router to forward packets. Applying game theory in such environments raises the following question: What are the incentives for nodes to cooperate, particularly when there may be natural disincentives such as higher energy consumption? Incentive mechanisms fall under this class. They are generally divided in two schemes: reputation-based systems and credit-based systems [3, 11]. In credit-based systems, cooperation is induced by means of payments received every time a node relays or forwards a packet, and such credit can later be used by these nodes to en- 


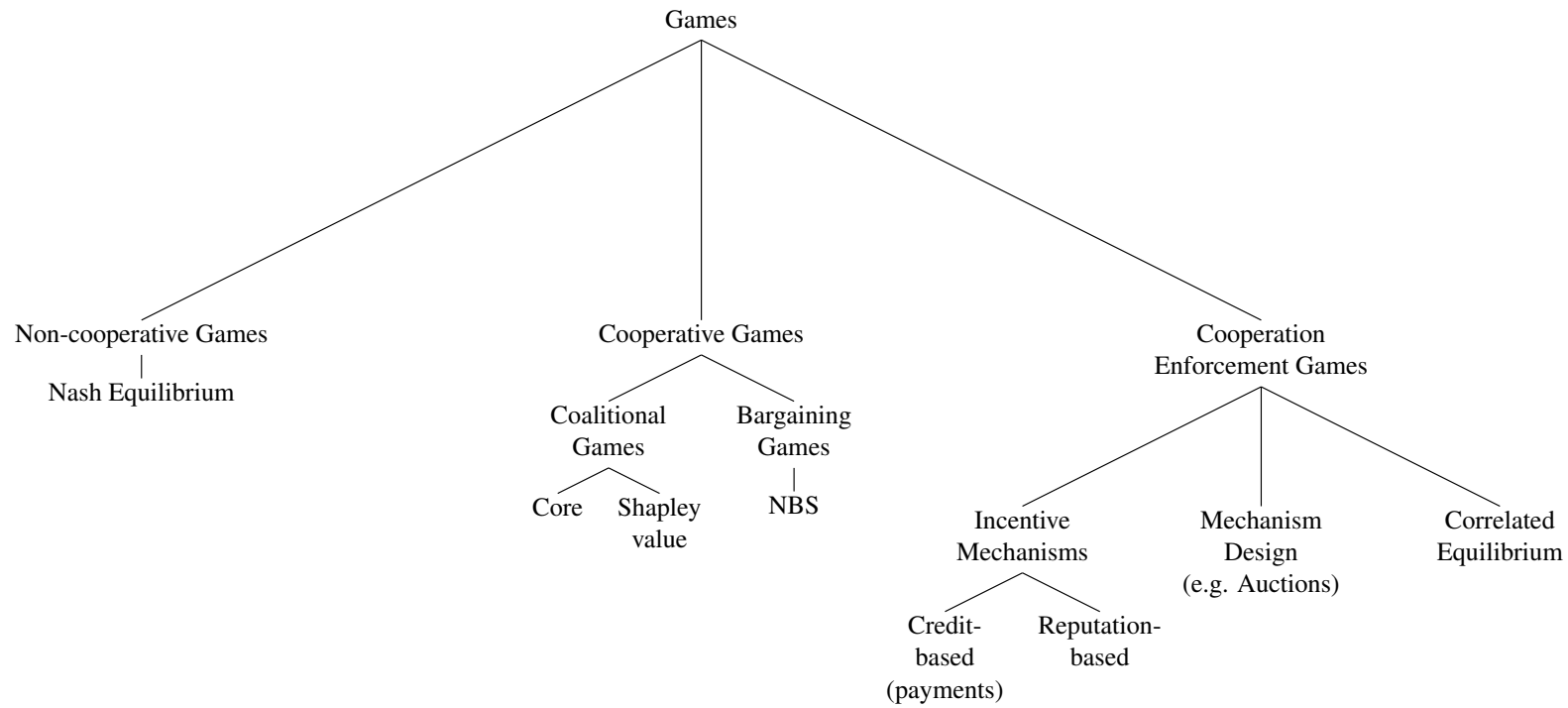

(a)
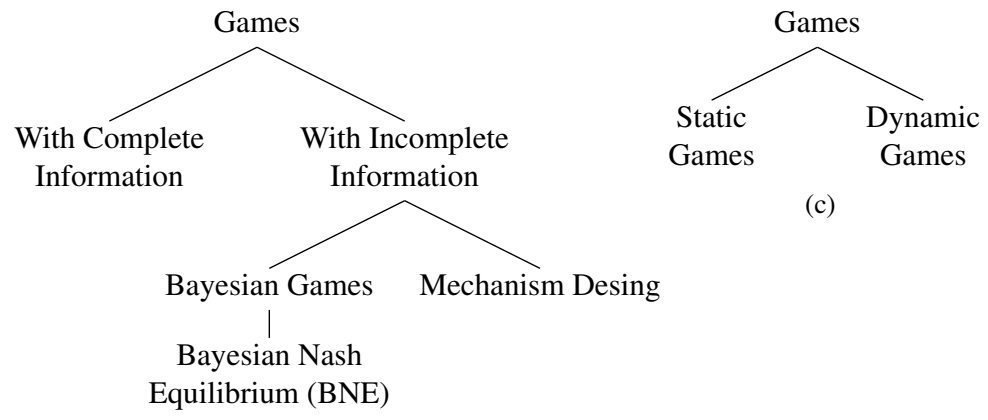

(c)

(b)

Figure 2: Taxonomy of games and the different methods to solve them, (a) cooperative, non-cooperative or cooperation enforcement games, (b) with complete information or with incomplete information, (c) static or dynamic games

courage others to cooperate. In reputation-based systems, each node assigns a reputation value to all other nodes it contacts with. As a node's reputation de-365 creases, its neighbors may refuse to perform services for it, leading to its gradual exclusion from the network. Nodes decide independently the extent of their cooperation with the network, trying to balance their reputation (too little cooperation might lead a node to $_{370}$ become an untrustworthy node) and resource considerations (too much cooperation may lead to a fast battery depletion).

Mechanism design [3, 5] is another branch which aims to enforce cooperation between nodes, and de-375 sign games that have dominant strategy solutions leading to a desirable outcome (either socially desirable, or desirable for the mechanism designer). The idea is to run an algorithm in an environment with multiple owners of resources. This algorithm takes into account the 380 preferences of the different owners. Indeed, the larger goal of mechanism design is often to design mechanisms in which the selfish behavior of players leads to such socially optimal outcome. Mechanism design could be with money (auctions), like Vickery-ClarkeGroves mechanisms [3], or without money, like House Allocation problem [3]. It is analogous to Bayesian games [3] in terms of privacy of owners information, but mechanism design makes the solution of a game much simpler.

Correlated equilibrium [16] is a solution method in which it is preferable for a player to follow an external correlation device, such as a trusted game coordinator. The traffic light example in [3] illustrates the idea. Imagine when two players drive up to the same intersection at the same time. If both attempt to cross, the result is a fatal traffic accident. In Nash equilibrium, players choose their strategies independently. In contrast, in a correlated equilibrium, a coordinator can choose the strategies for both players. For example, the coordinator can randomly let one of the two players cross with a certain probability. The player who is told to stop has a zero payoff, but he knows that attempting to cross will cause a traffic accident. Correlated equilibrium requires joint computation of strategies. In general, it is easier to computer those joint 
Table 1: Various game theory concepts and techniques used through the survey

\begin{tabular}{|ll|}
\hline Game Theory Concept & References \\
\hline NE-best response dynamics & {$[21],[24],[25],[26],[28],[76],[100],[115],[139]$} \\
\hline Repeated game & {$[21],[48],[50],[68],[96],[97],[98],[100],[105],[125],[157]$} \\
\hline Incomplete information & {$[21],[23],[69],[105],[113],[114],[136],[153],[154],[156]$} \\
\hline Bayesian NE & {$[23],[68],[113],[117]$} \\
\hline Dynamic game & {$[66],[69],[76],[113]$} \\
\hline Static game & {$[113]$} \\
\hline Mixed strategy NE & {$[49],[55],[57],[69],[136],[138],[139],[157]$} \\
\hline Pure strategy NE & {$[22],[76],[79],[125]$} \\
\hline Potential game & {$[28],[62],[100],[109],[112],[114],[116],[119]$} \\
\hline Shapley value & {$[31],[34],[67],[80],[81],[82]$} \\
\hline Core & {$[29],[32]$} \\
\hline Pareto optimality & {$[51],[109],[114]$} \\
\hline Nash Bargaining Solution $(\mathrm{NBS})$ & {$[51],[73],[104]$} \\
\hline Incentive mechanisms & {$[68],[69],[83],[105],[140],[141],[142],[153],[157]$} \\
\hline Mechanism design & {$[83],[85],[153],[154],[156]$} \\
\hline Cost sharing game & {$[80],[81]$} \\
\hline Extensive form & {$[69]$} \\
\hline Backward induction & {$[66],[158]$} \\
\hline Evolutionary game & {$[136],[139]$} \\
\hline
\end{tabular}

385 strategies, and finding a correlated equilibrium is polynomially solvable. However, finding an "optimal" cor-415 related equilibrium is computationally hard [3]. Besides, it needs a third party to observe and control the interaction of players.

\subsection{Other Classifications}

Game theory also classifies games according to other criteria, such that if games are static or dynamic. 420 In static games, we assume that there exists only one time step, which means that players move their strategy simultaneously without any knowledge of what other players are going to play. In dynamic games, players move their strategy in predetermined order, meaning ${ }_{425}$ that the move of one player is conditioned by the move of the previous players (i.e., the second mover knows the move of the first mover before making his decision).

Moreover, a game could also be classified into two 430 categories: a game with complete information and a game with incomplete information. In a complete information game, each player has all the knowledge about others' characteristics, strategy spaces, payoffs, and so forth, but all these information are not neces-43 sarily available in an incomplete information game. In games with incomplete information, the overhead resulting from information exchange is reduced, because each player predicts the strategies of other players. The resulting Nash equilibrium of this class of games is 440 usually called Bayesian Nash equilibrium (BNE) [3].
Table 1 contains an index that helps the reader to find where the different types of games and the various game theory concepts are used through the survey.

\section{Power Control and Medium Access Control (MAC)}

\subsection{Power Control}

The main sources of energy consumption in WSNs are sensing, processing and communication. Among them, communication is the one that consumes more energy. Hence transmission at optimal power level is of paramount importance. The optimal transmit power level is the power level which reduces the interference, increases the successful packet transmission and provides the desired QoS A large variety of schemes for power control issues in WSNs have been proposed. They have been surveyed in [17].

However, topology control solutions which use static transmission power, transmission range, and link quality, might not be effective in the real world. To address this issue, many distributed non-game theoretic algorithms have been proposed in the literature for dynamically adjusting transmission power level on each single node. In [18], a dynamic algorithm that considers the network lifetime as an essential metric using heuristics is proposed. A distributed algorithm that is based on geometric-programming for solving the power control problem is presented in [19]. A lightweight Adaptive Transmission Power 
Control (ATPC) algorithm for WSNs is proposed in [20]. In ATPC, each node builds a model for each of ${ }_{485}$ its neighbors, describing the correlation between trans445 mission power and link quality. This model employs a feedback-based transmission power control algorithm to dynamically maintain the link quality of individual links over time.

Game theory provides helpful distributed mecha450 nisms that allow for each single node to interact with its neighbors, and adjust its transmission power without the need of complete information about them. Table 2 lists the latest articles that use game theory in this $_{495}$ domain. The papers are summarized and discussed in the following subsections.

\subsubsection{Non-cooperative Games}

In [21], a non-cooperative game with incomplete in- ${ }^{500}$ formation is formulated to solve the distributed power control problem in WSNs. This proposal suggests 'not to transmit' at a certain game iteration when channel conditions are poor. The investigation for the existence of NE is done for two different cases, with fixed ${ }^{505}$ channel conditions, and with varying channel conditions using best response dynamics. It is observed that there exists a transmission power threshold and channel quality threshold that the nodes must comply with in order to achieve NE. Moreover, using re- ${ }^{510}$ peated games, nodes follow the transmission strategies to achieve NE even without presence of any third 470 party enforcement. A system that would allow only finite number of discrete power levels is considered. A metric called distortion factor is defined to investigate the performance of such system and compare it with systems that would allow any continuous power level.

475 This work also proposes a technique to find the power levels at which a node should transmit in order to maximize its utility and minimize the distortion. The results show that this algorithm achieves the best possible payoff/utility for the sensor nodes by consuming ${ }_{40}$ less power.

The power control game proposed in [22] is based on Code Division Multiple Access (CDMA) communication. It is observed that the cluster head $(\mathrm{CH})$ members structure in WSNs is similar to the base station terminal structure of CDMA. The difference here is that the utility function of the game considers the node's residual energy, and is given by:

$$
u_{i}\left(p_{i}, p_{-i}\right)=\mu \log _{2}\left(1+\operatorname{SINR}_{i}\right)-c\left(p_{i}\right)
$$

Both the pricing $c\left(p_{i}\right)$ and system model Signal Interference plus Noise Ratio $\left(\mathrm{SINR}_{i}\right)$ depends on the residual energy of node $\mathrm{i}$, as follows:

$u_{i}\left(p_{i}, p_{-i}\right)=\mu \log _{2}\left(1+G \frac{h_{i} p_{i} \frac{E_{\max }}{E_{i}}}{\sum_{j=1 j \neq i}^{N} h_{i} p_{i} \frac{E_{\max }}{E_{i}}+\delta^{2}}\right)-\lambda h_{i} p_{i} \frac{E_{\max }}{E_{i}}$ where $G$ is the gain of spread spectrum, $E_{i}$ and $E_{\max }$ are the residual energy and the maximum energy of node $i$, respectively, $\sum_{j=1}^{N}{ }_{j \neq i} h_{i} p_{i}$ is the sum of interferences node $i$ receives, $\delta^{2}$ denotes the thermal noise power of the channel, $\lambda$ is a dynamic adjust pricing factor, and $\mu$ is an income coefficient.

The existence and uniqueness of the NE is proved. Simulation results show that after considering the nodes' residual energy, path gain, and transmission power factors on the design of the pricing function, the performance of the power control game significantly improves, reducing the total transmission power efficiency, saving node energy and prolonging the network lifetime efficiently.

In [23], sensors are powered solely using renewable energy to replenish its stored energy. Each sensor considers its varying energy state as private information. The existence of $\mathrm{BNE}$ is proved. The BNE strategy of each sensor can be implemented using a threshold. If the energy state exceeds the energy threshold, the sensor transmits with a fixed power, otherwise, the sensor waits. This study shows how each sensor determines its threshold to maximize its utility function. The equilibrium of this Bayesian game model is compared with three different models: i) distributed perfect-information model, ii) a centralized system, and iii) a random-transmission model. The results show that the Bayesian model has a performance similar to the perfect-information model, but with a lower overhead, making the Bayesian model more suitable for real applications.

In a scenario with multiple sources and with multiple receiving clusters, all the sources send their information simultaneously towards the cluster heads. Simultaneous transmission causes interference at each receiving cluster which reduces the Signal Interference plus Noise Ratio SINR. Higher transmission power results in higher SINR, but increases the energy consumption and the interference to other receiving cluster heads. For this reason, a non-cooperative power control game is proposed in [24], where each sensor chooses its minimum transmission power independently to minimize its own cost in order to achieve a target SINR at the cluster head of the receiving cluster. The game can be expressed as the following cost minimization problem:

$$
\min _{P i} J_{i}\left(P_{i}, \gamma_{i}\right)=\min _{P i} b_{i} P_{i}+c_{i}\left(\gamma^{\mathrm{tar}}-\gamma_{i}\right)^{2}
$$
sion power (i.e., strategy values) for the $i$ th user, $P_{\max }$ is the maximum allowed power for transmission, and $J_{i}$ is the cost for player $i . b_{i}$ and $c_{i}$ are constant nonnegative weighting factors. $\gamma^{\text {tar }}$ is the target SINR at cluster $i$, and it is the same for all clusters. The existence of an equilibrium is proved, and the cost function has a minimum at the NE. Therefore, no player can 
Table 2: Proposals in WSN Power Control (Subsection 4.1)

\begin{tabular}{|c|c|c|c|c|}
\hline Article & Year & Class of Game & Game Techniques & Method of Energy Savings \\
\hline [21] & 2010 & Non-cooperative & $\begin{array}{l}\mathrm{NE} \text {, best response, repeated game } \\
\text { with incomplete information }\end{array}$ & $\begin{array}{l}\text { a distributed power control mechanism according to } \\
\text { different channel conditions }\end{array}$ \\
\hline$[22]$ & 2010 & Non-cooperative & $\mathrm{NE}$ & $\begin{array}{l}\text { the SINR model takes into account the residual energy } \\
\text { of nodes }\end{array}$ \\
\hline [23] & 2011 & Non-cooperative & $\begin{array}{l}\text { Bayesian Nash equilibrium (BNE), } \\
\text { incomplete information }\end{array}$ & $\begin{array}{l}\text { an energy efficient power control with reduced over- } \\
\text { head }\end{array}$ \\
\hline$[24]$ & 2012 & Non-cooperative & $\mathrm{NE}$, best response & $\begin{array}{l}\text { optimization of the transmission power independently } \\
\text { to achieve a target SINR }\end{array}$ \\
\hline$[25]$ & 2012 & Non-cooperative & $\mathrm{NE}$, best response & $\begin{array}{l}\text { a game with an energy efficient convergence algorithm } \\
\text { (NPC) to converge quickly to the NE point }\end{array}$ \\
\hline$[26]$ & 2012 & Non-cooperative & $\mathrm{NE}$, best response & $\begin{array}{l}\text { the computational and sensing energy are taken into } \\
\text { account in the energy consumption model }\end{array}$ \\
\hline$[28]$ & 2014 & Non-cooperative & $\begin{array}{l}\text { Ordinal Potential Game, best re- } \\
\text { sponse }\end{array}$ & $\begin{array}{l}\text { a joint channel allocation and power control game to } \\
\text { reduce both the energy consumption and the interfer- } \\
\text { ence }\end{array}$ \\
\hline [29] & 2008 & Cooperative & $\begin{array}{l}\text { coalition, core, repeated game, De- } \\
\text { ferred Acceptance Procedure (DAP) }\end{array}$ & $\begin{array}{l}\text { maximizing the number of bits that can be transmitted } \\
\text { per Watt of the consumed power }\end{array}$ \\
\hline$[31]$ & 2010 & Cooperative & coalition, Shapley value & $\begin{array}{l}\text { a fair allocation of power among collaborating nodes } \\
\text { in fusion center based WSNs }\end{array}$ \\
\hline$[32]$ & 2011 & Cooperative & coalition, core & $\begin{array}{l}\text { optimizing the transmission power of a group, and } \\
\text { choosing the best group in terms of power conserva- } \\
\text { tion }\end{array}$ \\
\hline$[34]$ & 2013 & Cooperative & coalition, Shapley & $\begin{array}{l}\text { a fair distribution of the total cooperative cost among } \\
\text { sources, and trade-off between energy efficiency and } \\
\text { end-to-end delay }\end{array}$ \\
\hline
\end{tabular}

benefit by deviating from the NE. Bounds on source power at NE are also proved. Performance analysis

is done using computer simulation, which illustrates the influence of interference and distance between the source and the target cluster on the NE. Finding Pareto optimality is considered a potential future investigation for this proposal.

The work presented in [25] claims that previous works on power control in WSNs did not focus on the system convergence. From this perspective, this proposal focuses on constructing a non-cooperative game model with a convergence algorithm, called NPC, that guides nodes to converge quickly to the $\mathrm{NE}$ point. Power consumption is taken as the cost for the game model. The game is decentralized and the information of each node can not be known by the others. Therefore, the best response choice is used to achieve the NE. Then, the system convergence to the NE point is evaluated and guaranteed using the NPC algorithm. The NPC algorithm shows a remarkable optimization in energy efficiency and convergence speed, without accessing the profile of the others.

In [26], a Game theory based Energy-efficient Power control Strategy (GEPS) is proposed for cognitive sensor networks. In cognitive sensor networks, unlicensed users (secondary) share a common spectrum with li-565 censed users (primary). Each user wants to maximize its utility function under interference temperature con- straints as follows:

$$
\begin{gathered}
\max _{p_{i}} \frac{\mathrm{B} \log \left(1+\mathrm{SINR}_{i}\right)}{p_{i}+\alpha} \\
\text { s.t. } \quad \sum_{i=1}^{n} h_{i} p_{i} \leq \mathrm{M}
\end{gathered}
$$

where $p_{i}$ is the power allocation of user $i, \mathrm{~B}$ is the available wireless spectrum bandwidth, $M$ is the interference temperature limit, which is described as a threshold of total received power at the primary user. $h_{i}$ is the link gain between a secondary user $i$ and the primary user. Both the computational and sensing energy, $\alpha$, are taken into account in the energy consumption model. Simulations are done for testing the energy efficiency of the power control game. It can be found that under some conditions this game is a supermodular game [3], which means it has good convergence properties to the NE point using the best response algorithm. GEPS outperforms the Game-based QoS-oriented Power allocation Strategy (GQPS) [27] in terms of energy efficiency and system utility (i.e., 50 the average value of cognitive users' utilities). However, as the number of users increases, the system utility in GEPS decreases dramatically, while it keeps nearly unchanged in GOPS.

A Joint Channel Allocation and Power Control Game Algorithm (JCAPGA) is proposed in [28]. Power control is used to reduce the network energy consumption, and channel allocation is used to reduce 
the network interference (generated by other transmit-595 ting and receiving nodes). However, selecting different channels affects the node power. Therefore, this paper aims to optimize the network performance through collaboratively controlling the two techniques. Nodes' transmission power, the network interference, and the 600 residual energy of nodes are taken as the model paramlower transmission power. The model is proved to be an OPG in order to ensure NE. The best response strategy is considered to improve the convergence speed.605 Simulation results show that, in JCAPGA the channel allocation is uniform, the network interference is low, and the energy consumption is balanced.

\subsubsection{Cooperative Games}

\section{0}

A repeated coalitional game is presented in [29]. The motivation is that each node can likely obtain better utility by forming groups and controlling its power cooperatively rather than individually. Nodes compete with the others trying to enhance their own power ef-615 ficiency subject to QoS constraints. In this work, it is preferable to maximize the number of bits that can be transmitted per Watt of the consumed power with respect to SINR, rather than to maximize the throughput, according to the definition of power efficiency620 [30]. The problem is modeled as two-sided one-to-one matching game, in which an owner is matched with a single non-owner in order to help the non-owner to achieve improved power efficiency. Then, the game is repeated using the Deferred Acceptance Procedure625 DAP [3]. This technique produces a single matching in the core at each repetition until reaching a certain "stable" state. The optimal power efficiency is computed in each step via a non-linear optimization problem, as the following:

$$
\max u_{i}=\frac{r_{i}}{\sum_{s \in S} p_{i, s}}
$$

Subject to:

$$
\begin{aligned}
\sum_{s \in S} p_{i s} & \leq P_{i}^{\max } \text { for all } i \in \mathcal{N} \\
T_{i} & \leq T_{i}^{\max } \text { for all } i \in \mathcal{V} \\
\lambda_{i} & \leq r_{i} / \Lambda_{i} \text { for all } i \in \mathcal{V}
\end{aligned}
$$

with $r_{i}=\mathrm{B} \sum_{s \in S} \log _{2}\left(1+\mathrm{SINR}_{i, s}\right)$, where $r_{i}$ is the transmission rate at sensor $i$, and $B$ is the bandwidth of each subcarrier. The parameters are: the set of sensors $(\mathcal{V})$, the set of idle subcarriers $(\mathcal{S})$ that are detected as not allocated to primary users, the transmission power constraint of sensor $i\left(P_{i}^{\max }\right)$, and the transmission delay 645 constraint of sensor $i\left(T_{i}^{\max }\right)$. The variables are: sensor $i$ 's transmission power over subcarrier $s\left(p_{i, s}\right)$, and the average transmission delay $\left(T_{i}\right)$, including queueing delay per packet considering an $M / M / 1$ queue. Simulation results show that the matching in the core im-650 proves the total power efficiency more than the social optimal, though it is less fair.

In [31], tools from cooperative game theory are used to develop a formal analytical framework for fair allocation of power among collaborating nodes in a Fusion Center (FC) based WSNs. The goal is to achieve a sequential estimation task, while at the same time maximizing the overall network lifetime. The concept of the Shapley value is used to achieve a fair power allocation among distributed nodes. Simulation results show that the proposed solution achieves the target estimation quality at the FC, and at the same time the lifetime of the overall sensor network is increased.

The proposed algorithm allows nodes to choose the best group in their coverage area in terms of power conservation using coalitional game theory.

A cooperative game is proposed in [32] to optimize data transmission of a group (not a single node) by forming coalitions. A mobile node may move to a new location to join a desirable group. It requests the group leader first. Then, the group leader evaluates the benefit of group membership for the node using coalitional game theory. If the membership is beneficial, the leader sends an invitation to the node. The node itself may receive many invitations and can choose the best group in their coverage area by comparing its own utility with the offered one. The correctness of the proposed protocol is proved by searching for failures in it, through evaluating all possible behaviors of sensors using the Maude tool [33]. The work proves that the core is not empty and simulation results show that any node could always save more energy by joining a group.

In [34], a power control solution based on the tradeoff between energy efficiency and end-to-end delay is presented. A cooperative coalitional game is proposed to obtain a power control solution that achieves a fair distribution of the total cost among sources. It is observed that the additional energy cost function and the delay cost function are continuously differentiable (i.e., minimizing the delay is achieved by minimizing to minimize its utility function of discounted sum of transmission power increase cost and source-to-sink delay cost. Shapley value is used as a solution of the cooperative allocation game. The results illustrate the impact of delay and energy cost parameters on the energy consumption associated to different coalitions. They show that selecting a larger coalition is better than a smaller one in cooperative games.

\subsubsection{Discussion and Future Directions}

From the previously reviewed proposals, we notice that non-cooperative games have been preferably used for energy efficiency in power control problems. This is because sensor nodes do not use any information about the separate transmission power level strategies taken by other nodes, which means that control packets 
are greatly reduced (see [21, 23, 25]). There are different opinions about: i) which class of games and solution concepts are more appropriate, ii) how to save energy, iii) and how to converge to a stable NE point (see 710 C of proposals take into account the channel conditions to save energy [21], while others like [22] consider nodes' residual energy in the utility function. However, proposals like [29] say that it is more important $7_{715}$ per Watt of the consumed power, while [26] takes computational and sensing energy also into account in the energy model. A collaborative control of both channel allocation and power control is taken into account in ${ }_{720}$ 665 [28]. In [24], a cluster-based WSN with multiple receiving clusters is studied. [32] cares about optimizing data transmissions of a group. The trade-off between energy efficiency and end-to-end delay are taken into account in [34, 29]. [34] achieves a fair distribution of 725 670 the total cooperative cost among sources. It is worth noting that not all the proposals evaluate the performance of their proposed solutions or algorithms. Only [32, 21] do.

As a conclusion, we notice that there is not one sin-730 675 gle strategy or one class of games which is considered a general solution for saving energy in this domain. The applied mechanism depends on different factors like the network scenario, if it is applicable in real cases or not, and QoS constraints, among others. In the future work, game theory models could be applied ${ }_{735}$ to address the issues of power control mechanisms in energy harvesting WSNs.

\subsection{MAC}

Reducing energy consumption is a challenge when ${ }_{740}$ designing a MAC protocol for WSNs. Idle listening, collision, and overhearing are the major sources of energy consumption in a WSN [35].

Recent proposals in this domain take the energy efficiency of a node as the main goal to solve. For exam-745 ple, in order to achieve low power operation in random access MAC schemes, X-MAC [36] introduces a series of short preamble packets, instead of one long preamble as in B-MAC [37]. In synchronized schemes, TMAC [38] allows an adaptive length of the active peri-750 ods instead of fixed sleep/active cycles as proposed in S-MAC [39]. L-MAC [40] is a Time Division Multiple Access (TDMA)-based scheme that organizes time into frames and eliminates the channel access by precisely scheduling who is allowed to transmit in each 75 slot. Z-MAC [41] is a hybrid MAC protocol that combines some of the best features of the TDMA and Carrier Sense Multiple Access (CSMA). Z-MAC improves energy efficiency by achieving high channel utilization and enhancing contention resolution. Compre-760 hensive studies which analyze and compare the different MAC protocols for WSNs are presented in [35, 42].
Finally, LWT-MAC [43] is an adaptive MAC protocol suitable to be enhanced using game theory approaches. It combines an unscheduled channel access, based on low power listening, with an opportunistic scheduled wake up after transmissions mechanism.

It is clear that each node has a direct influence on its neighboring nodes while accessing the channel. These interactions between nodes lead researchers to introduce the concept of game theory in the design of MAC protocols in order to improve energy efficiency as well as the end-to-end delay in a decentralized manner. Game theory has been applied in contention free schemes, showing that it could save energy by decreasing collisions (e.g., Multiple Access Game [44], [45]). In the following subsections, we present and discuss the latest contributions in MAC schemes using game theory for energy savings purposes. The considered proposals in this survey are summarized in Table 3 Notice that game theory has been also considered to prevent node's misbehavior at MAC layer. For instance, in CSMA/CA MAC protocols, some nodes may use different backoff parameters to get more bandwidth than the other honest contenders [46, 47]. However, since those papers do not focus on energy efficiency issues, we we do not overview them in details.

\subsubsection{Non-cooperative Games}

A simplified Game-theoretic Constraint Optimization scheme (G-ConOpt) is presented in [48], in which its performance is optimized in an energy efficiency manner. In G-ConOpt, time is divided into superframes and every super-frame has two parts: an active part and a sleeping part. During the active part, each node plays a game and contends for the channel. During the sleeping part, each node turns off its radio to preserve energy. The durations of the active and sleeping parts are adjusted according to the estimated game state too. Firstly, a node estimates the current state of the game, defined as the number of its active opponents $n$. Secondly, the node adjusts its equilibrium strategy, the minimum Contention Window (CWmin), to the estimated number of its opponents $n$. It is not needed to compute the optimal value of $\mathrm{CWmin}$. The final value of $\mathrm{CW}$ is the optimal one, and the best strategy for a player is to set $\mathrm{CWmin}=\mathrm{CW} / 2$. That is to say, GConOpt would not cause any more energy consumption. Power consumption and energy efficiency of GConOpt is compared with S-MAC and CSMA by simulation. Results indicate that the power consumption in both S-MAC and CSMA is almost constant, and increases adaptively in GConOpt with the increasing of the traffic loads. Energy efficiency in GConOpt is higher than in S-MAC and CSMA.

In [49], a MAC scheme based on p-persistent slotted ALOHA and constructed a simple non-cooperative game is proposed in order to determine the value of the channel attempt probability $p$. Firstly, general- 
Table 3: Proposals in WSN Medium Access Control (MAC) (Subsection 4.2)

\begin{tabular}{|llllll|}
\hline Article & Year & Class of Game & Scheme & Game Techniques & Method of Energy Savings \\
\hline$[48]$ & 2009 & Non-cooperative & $\begin{array}{l}\text { mixed } \\
\text { (scheduling } \begin{array}{r}\text { based } \\
\text { contention) }\end{array}\end{array}$ & $\begin{array}{l}\text { history-based estima- } \\
\text { tion, infinitely repeated }\end{array}$ & $\begin{array}{l}\text { adjusting the transmission probability and } \\
\text { the sleeping time of a node according to the } \\
\text { number of its opponents }\end{array}$ \\
\hline$[49]$ & 2010 & Non-cooperative & $\begin{array}{l}\text { contention based } \\
\text { mixed strategy NE }\end{array}$ & $\begin{array}{l}\text { optimizing the channel attempt probability } \\
\text { taking energy consumption into account }\end{array}$ \\
\hline$[50]$ & 2013 & Non-cooperative & scheduling based & NE, repeated game & $\begin{array}{l}\text { optimizing the sleep interval between con- } \\
\text { secutive wake-ups (duty cycle control) }\end{array}$ \\
\hline$[51]$ & 2007 & Cooperative & scheduling based & $\begin{array}{l}\text { NE, Pareto optimality, } \\
\text { bargaining game }\end{array}$ & $\begin{array}{l}\text { determining the optimal sleep and wakeup } \\
\text { probabilities }\end{array}$ \\
\hline
\end{tabular}

ized payoffs were designed to reflect the costs of energy consumption and throughput deterioration. Next, $\mathrm{NE}$ is found in a closed form. Each player then has only two pure strategies: to always attempt to deliver a packet, and to never attempt it. However, the NE is the mixed one. Using the NE, the effect of the payoffs on the stability of a sensor network is investigated. It is observed that the set of feasible values of the payoff for saving energy shrinks as the traffic load per leaf node increases.

It is observed that the higher/lower the duty cycle, the higher/lower the energy consumption at receiving nodes, and the lower/higher the energy consumption 775 at transmitting nodes are. Thus, a Non-cooperative Duty-cycle Control Game (R-NDCG) for reducing the idle-listening time is proposed in [50]. It aims at optimizing the sleep interval between consecutive wakeups for random asynchronous wakeup MAC protocols.780 In this MAC scheme, the sender waits for a beacon signal from the receiver before starting to transmit. Since each sender receives beacon signals from several nodes, the data are routed on multiple paths. In this context, an optimization framework for minimizing the energy waste of the most power-hungry node is pre-785 sented. Firstly an analytic model that predicts nodes' energy consumption is derived. Then, the model is used as a part of the optimization process. At the transmitter, the transmission energy model represents the sum of the energy spent to deliver data packets, and 790 the energy spent to listen to the channel before a beacon signal is received and the contention is won. At the receiver, it is the sum of the total energy consumption due to data packet reception, the energy spent for beacon signal transmission, and the amount of energy 795 consumed between the generation of the beacon signal and the reception of the packet. The objective function, the minimization of energy waste of the most powerhungry node, contains sums and products of rational terms. Thus, it is neither linear nor convex. Therefore 800 a multi-start local search is presented first for solving the problem. The obtained solution was considered as a comparison benchmark for assessing the overall performance of the game theoretic approach. Then, a game theory based solution is proposed as follows: Let
$G=\left[\{\mathcal{N}\},\{\mathcal{R}\},\left\{C_{s}^{(i)}().\right\}\right]$ denote the NDCG, where $\mathcal{N}$ is the set of the nodes, $\mathcal{R}$ is the set of strategies, and $C_{s}^{(i)}($. is the cost function of user $i$. Each user $i$ selects a beacon rate $r_{s}^{(i)} \in R$, which corresponds to the outcome of the game in terms of selected beacon signal rate (i.e., the duty-cycle). In the NDCG, each user minimizes his own cost function in a distributed way. Formally, the NDCG game can be expressed as:

$$
\arg \min \left[C_{s}^{i} r_{s}^{(i)}\right] ; \forall u_{s}^{(i)} \in \mathcal{N}
$$

The cost function of the proposed game represents a trade-off between a node's energy and the energy of any node belonging to a set of nodes producing data traffic towards node $i$. Since users act selfishly, the equilibrium point is not necessarily the best operating point from a social point of view. However, it is proved via simulations that the NE of the distributed game achieves a desirable result.

\subsubsection{Cooperative Games}

In [51], an optimal energy savings mechanism for a sensor node is presented. It uses a sleep and wakeup strategy for energy conservation. The node switches to sleep mode if channel quality is bad and switches to the active mode, from the listen and sleep modes, with probability $P_{\text {active, listen }}$ and $P_{\text {active,sleep }}$, respectively, at the beginning of periodic time intervals. The strategy for the first player is to select $P_{\text {active,sleep. For the second }}$ player the strategy is to select $P_{\text {active,listen. Payoff for }}$ the first player is the packet blocking probability $P_{\text {block }}$ (due to the sleep mode), and for the second player the packet dropping probability $P_{\text {drop }}$ (due to buffer overflow). This strategy results in a trade-off between $P_{\text {drop }}$ and $P_{\text {block }}$. A bargaining game is formulated to determine those probabilities under energy constraints. A NE, which is Pareto optimal, is obtained as the solution of this game in order to obtain the optimal sleep and wakeup probabilities. The solution basically eliminates the selfishness of nodes that try to conserve energy at the expense of high $P_{\text {block}}$.

\subsubsection{Discussion and Future Directions}

We can infer from the presented proposals and Table 3 that game theory has been applied to address the 
energy efficiency in different MAC schemes, scheduling based [50, 51], contention based [49], and mixed 860 (scheduling and contention based) [48]. Although the number of proposals in this domain is limited, we believe that they are sufficient to break the ice in this domain. In a contention based scheme [49], a noncooperative game is used to determine the value of 865 the attempt probability $p$. Most of the schedulingbased MAC schemes focus on the optimization of the active and the sleeping intervals, which helps in saving energy and increasing the network lifetime (see [48, 50, 51]).

We mention that non-cooperative games are preferably used. Only one proposal uses cooperative games with a Nash bargaining solution. However many issues still need to be addressed in this domain. For example, the use of game theory for energy efficiency 875 in contention free MAC schemes (e.g., TDMA, Hybrid MAC schemes, high data rate applications, and multi-channel MAC protocols are recommended for future work. Moreover, game theory tools are suitable to solve some problems in receiver initiated $\mathrm{MAC}_{880}$ protocols. For example, in this class of MAC protocols the sender waits for a beacon from its intended receiver before starting the transmission of data. If the time between sending two consecutive beacons increases (i.e., the receiver is saving energy), the proba-885 bility that many senders will send to the same receiver and collide will also increase, which affects the energy level of other nodes in the network. Game theory provides tools to solve this trade-off problem and achieve both a social and a local optimum solution at the same 890 time. Game theory may also be suitable for cross layer designs (e.g., to investigate how the design of MAC layer affects the network layer).

\section{Routing and Clustering}

\subsection{Routing}

Routing refers to determining a path for a message from a source node to a destination node. The routing problem is an attractive research area in WSNs. Generally, when attempting to optimize this problem, a lot of metrics should be taken into consideration. For modeling the cost of a transmission, some parameters at each node are considered. For example, the distance (i.e., delay and power consumption are proportional to it), remaining energy, and transmission rate of each link, etc. Thus, a good candidate model should take these parameters into account and consider the distributed nature of WSNs.

Since most of the routing protocols developed for wired networks pursue the attainment of high QoS, they are impractical in WSNs. Thus, different nongame theory approaches have been done for energy 895 aware routing in WSNs. Computational Intelligence
(CI) based approaches have been widely applied in the domain of energy aware routing. Such approaches are usually based on Reinforcement Learning (RL), Swarm Intelligence (SW), Genetic Algorithms (GAs) or Neural Networks (NNs). These approaches are surveyed and briefly explained in [52, 53]. However, these approaches are generally based on metaheuristics which do not necessarily converge to an exact or an optimal result, and are usually centralized (except reinforcement learning, see [53]). Besides, an offline learning phase, like GAs or offline NNs, can neither cope with changing properties of the network, nor provide an energy efficient routing scheme. Ant-based routing is a flexible technique, but generates a lot of additional traffic because of the forward and backward ants moving through the network.

Game theory has been successfully applied to different WSNs' routing and load balancing problems that consider energy efficiency and network lifetime maximization as main goals. Issues such as the presence of selfish nodes in a network have been analyzed using game theory based, for instance, on incentive mechanisms. The idea behind these models is that for each successfully delivered data packet, the destination node pays a credit or modify the reputation of the source in all intermediate nodes that participate in the routing game. However, each data packet transmission has a cost for each node that participates in the route. This cost is a function of the three previously mentioned parameters. Nodes -wanting to maximize their profit- will accept to be part of the path if its profit is not negative [54].

In this subsection, we summarize the latest contributions in this domain. The considered proposals are summarized in Table 4

\subsubsection{Non-cooperative Games}

In [55], a Game Theoretic distributed Energy Balance Routing (GTEBR) algorithm is proposed to prolong the network lifetime. It allows a node to make decisions whether to take part in the routes by considering its residual energy and other factors in order to make the whole network's energy consumption more balanced. In this game, each node's strategy space is $S_{i}=\{0,1\}$, the value 0 means that a node $i$ chooses the strategy not relaying the data from its former hop node, and the value 1 means the opposite. A sensor node is modeled as having a mixed strategy, which means that a node can transmit the data with probability $p_{T}$ and be silent with probability $1-p_{T}$. The probability $p_{T}$ is defined as a function of the residual energy $E_{r, i}$ and actual payoff $P_{a, i}$ :

$$
p_{T i}\left(P_{a, i}, E_{r, i}\right)
$$

The existence of NE is proved and the algorithm is compared with Maximum Energy Minimum Hops 
Table 4: Proposals in WSN Routing (Section 5.1)

\begin{tabular}{|c|c|c|c|c|c|}
\hline Article & Year & Algorithm & Game Class & Game Techniques & Method of Energy Savings \\
\hline [55] & 2007 & GTEBR & Non-cooperative & mixed strategy NE & $\begin{array}{l}\text { helping nodes to make decisions whether to take } \\
\text { part in the routes according to their residual en- } \\
\text { ergy }\end{array}$ \\
\hline [57] & 2009 & VGTR & Non-cooperative & $\begin{array}{l}\text { mixed strategy NE, action re- } \\
\text { sults awareness }\end{array}$ & $\begin{array}{l}\text { an energy efficient of Data Routing Tree (DRT) } \\
\text { construction, minimize network segmentation, ro- } \\
\text { tate the selection of a node's next hop }\end{array}$ \\
\hline [62] & 2010 & DSR-G & Non-cooperative & ordinal potential game & $\begin{array}{l}\text { choosing reliable routes that prolong the network } \\
\text { lifetime }\end{array}$ \\
\hline [64] & 2011 & HDBR & Non-cooperative & $\begin{array}{l}\text { Stackelberg model, local infor- } \\
\text { mation }\end{array}$ & constructing energy balanced routing trees \\
\hline [66] & 2012 & SGEAR & Non-cooperative & $\begin{array}{l}\text { dynamic game, backward in- } \\
\text { duction }\end{array}$ & $\begin{array}{l}\text { taking the residual energy of nodes and the energy } \\
\text { consumption of paths into consideration }\end{array}$ \\
\hline [67] & 2011 & - & Cooperative & $\begin{array}{l}\text { coalition, absorption coeffi- } \\
\text { cient, Shapley value }\end{array}$ & $\begin{array}{l}\text { considering the proportion of sent data and the } \\
\text { proportion of forwarded data when making deci- } \\
\text { sions }\end{array}$ \\
\hline [68] & 2008 & - & Cooperation Enforcement & $\begin{array}{l}\text { infinite repeated game, incen- } \\
\text { tive mechanism (reputation- } \\
\text { based) }\end{array}$ & $\begin{array}{l}\text { packets transmission efficiency is taken into ac- } \\
\text { count when a node decide whether to forward } \\
\text { packets or not. }\end{array}$ \\
\hline [69] & 2009 & - & Cooperation Enforcement & $\begin{array}{l}\text { dynamic Bayesian game, in- } \\
\text { complete info, incentive mech- } \\
\text { anism (credit-based), extensive } \\
\text { form, pure and mixed BNE }\end{array}$ & helping nodes to decide energy-aware paths \\
\hline
\end{tabular}

Routing (MEMHR) [56]. Simulation results show that the residual energy distribution is higher and the network lifetime is longer in GTEBR than in MEMHR. However, GTEBR exhibits a higher average hops which increases the delay.

In [57], game theory is used for efficiently constructing a Data Routing Tree (DRT) with the aim to prolong the lifetime of the entire WSN by minimizing the network segmentation. The resulting protocol is called Versatile Game Theoretic Routing Protocol (VGTR). In the protocol the energy is expressed differently. Instead of expressing it with an absolute representation (i.e., using Joules), a time derivative representing the amount of remaining lifetime is used based on past workload. The algorithm induces an energy-aware and efficient collaborative behavior to the nodes. The nodes predict the results of their actions and rotate the selection of their next hop in a calculated way. The rotation is achieved using the payoff function. A node will assign a high probability to a neighboring node (i.e., next hop) that will extend its life time. The performance of VGTR in terms of energy consumption outperforms Directed Diffusion (DD) [58], and Sim-

920 ple Energy Efficiency Routing (SEER) [59]. Both Energy Aware Routing (EAR) [60] and VGTR attempt to balance the load between multiple paths. In addition, ${ }_{930}$ VGTR nodes are able to detect hot-paths and critical nodes and to minimize their utilization. In comparison with Low-Energy Adaptive Clustering Hierarchy (LEACH) [61], VGTR has a higher rate of node deaths which means that LEACH outperforms VGTR in pro- ${ }_{935}$ longing the network lifetime.

In [62], a reliable routing model against selfish nodes is proposed. The nodes should choose reliable routes that prolong the network lifetime. When the distance between a node and the sink is fixed, the node should transmit to a distant neighbor in order to save total network energy. Moreover, sending to a closer neighbor, increases the total number of hops to the sink, which also affects the reliability. For improving the reliability of transmission, shorter paths are preferred. However, it creates some hot areas in which nodes die quickly. To solve this contradiction, game theory is used. Besides, the NE point is reached after proving that the game is an OPG The utility function proposed considers four factors. It is defined as follows:

$$
u_{i}(p)=c_{i}(p)+r_{i}(p)-p_{i}(p)-h_{i}(p)
$$

where $h_{i}(p)$ is the collision utility, $p_{i}(p)$ is transmission power, and $c_{i}(p)$ is the connectivity utility:

$$
c_{i}(p)=c_{i}\left(p_{i}, p_{-i}\right)=\left(1-f\left(\operatorname{area}_{n}\right)\right) / f(\text { area })
$$

where $f\left(\operatorname{area}_{n}\right)$ is the area of the free region, $f$ (area) is the whole monitoring area of the sensor network, and $r_{i}(p)$ is the reliability utility:

$$
r_{i}(p)=r_{i}\left(p_{i}, p_{-i}\right)=N b r_{i}-1 / D_{i}
$$

where $N_{b r}\left(p_{i}\right)$ is the number of node $i$ 's neighbors within transmission power $p_{i}$, and $D_{i}$ is the probability of dropping packets. This model is applied over Dynamic Source Routing (DSR) protocol [63] and named as DSR-G. Results show that after applying game theory the selfish nodes have less impact in DSR-G than DSR.

In [64], Heterogeneous Balanced Data Routing (HDBR) is presented. It is a game theoretical distributed algorithm aiming to construct energy balanced 
routing trees in heterogeneous WSNs. It considers nodes with parent role are leaders of the game and nodes with child role are followers. Utility functions use local information of nodes. Parents have cooperative behavior, while children have selfish behavior trying to gain more individual utility. Leaders make decisions before the followers, since they can predict the followers' decisions. The behavior of the parents influences the behavior of the children, so that even with selfish actions of children as followers, they still contribute to the global benefit of the game (i.e., constructing a balanced tree for the entire network). Parents also try to decrease the load of other adjacent parents which are two hop away nodes at the same level. HDBR not only considers the amount of data each node has to transmit, but also bandwidth and delay as balancing criteria. HBDR outperforms the cumulative algorithm,995 proposed in [65], in prolonging the lifetime of WSNs. However, such a proposal still need to be compared with other protocols in order to make a more accurate evaluation.

A Sub-Game Energy Aware Routing (SGEAR) is 000 presented in [66]. The scheme is based on the fact that the optimization problem of routing could be mapped into a dynamic game problem, and thus, could be solved using Backward Induction method [4]. SGEAR takes the residual energy of the nodes and the energyoos consumption of the path into consideration. Compared with energy-aware routing, SGEAR can provide stable routes and optimize energy consumption of the whole network. Moreover, the algorithm is suitable for being combined with scheduling based scheme for prolong ${ }_{\top 010}$ ing the lifetime of WSNs.

\subsubsection{Cooperative Games}

In [67], a data transfer strategy is proposed to recoalitions. The idea is to consider "the proportion of sent data and the proportion of forwarded data". The coalitions are formed based on a Markov process. The concept of absorption coefficient is proposed to meais used to share the coalitions' payoff. NE is used here to determine the coalitions' approximate data transfer strategies of the formed coalitions. However, finding the exact NE in this proposal is a NP problem. Thus,

985 a genetic algorithm is given to calculate it approxi+o25 mately. Finally, the energy consumption of nodes in both when they work alone and when they cooperate is compared. Simulation results show that nodes consume less energy when they cooperate.

\subsubsection{Cooperation Enforcement Games}

A self-learning repeated-game for cooperation enforcement between randomly deployed nodes with local information only is proposed in [68]. This frame- work is applied in the case that nodes may not know how to cooperate even if they are willing to do so. The node's utility is quantified as its own packet transmission efficiency, which is defined as the ratio of the power for successful self-generated traffic over the total power used for self-generated traffic and packets forwarding. The goal of the node is to maximize the long-term average efficiency. The stage utility function for node $i$ can be represented as:

$$
U^{(i)}\left(a_{i}, a_{-i}\right)=\frac{P_{\mathrm{s}, \mathrm{good}}^{(i)}}{P_{s}^{(i)}+P_{f}^{(i)}}
$$

where $a_{i}$ is node $i$ 's packet forwarding probability, $a_{-i}$ is the other nodes' forwarding probability, $P_{\mathrm{s}, \mathrm{good}}$ is the power consumed in successful (good) transmission of node $i$ own packets to its destination, $P_{f}$ is the power consumed in forwarding other nodes' packets, and $P_{S}$ is the power consumed in transmitting node $i$ own packets. In this game the nodes/players do not know when the game ends (i.e., infinitely repeated game). Unlike the one-time game, a repeated game allows a strategy to be related to the past moves and results in reputation and retribution effects. Therefore, any cooperative equilibrium that is more efficient than the NE of the one stage game can be sustained, and any deviation causes a punishment from other nodes in the future. The second step utilizes a learning algorithm to achieve the desired efficient cooperative equilibrium. The two proposed steps are applied iteratively until no more efficient cooperation point can be achieved. The proposed game is able to enforce cooperation among selfish nodes. Nodes will not have incentive to lie because lying nodes will be detected using majority voting.

In [69], the source/forwarder problem is formulated as a dynamic Bayesian game with incomplete information. This game is played by every node participating in the packet delivery, thereby helping the nodes to decide energy-aware paths toward a sink. The factors, such as energy, location (related to mobility), and cooperation between sensors, are taken into account in this work. In addition, each sensor is unaware of the energy state of its neighboring sensors. The update system is based on Bayesian game theory. It improves the efficiency of path selection and minimizes the need of instantaneous updates about local sensors' energy.

A two-player Bayesian game is modeled. One player is a sensor node, denoted by $i$ (source). The other player is a one-hop neighbor $j$ of the source $i$. In Fig. 3, $N$ represents an entity the decides $j$ 's type. Source $i$ with the belief $B_{0}$ that forwarder $j$ 's energy level is sufficient has two pure strategies: forwarding $H$ packets, or discarding the packets and remain in idle mode. The work proves that the strategy combination ( $i$ plays "sleep mode" if $j$ is energy constrained but plays "send $H$ packets" if $j$ has sufficient energy, $j$ 


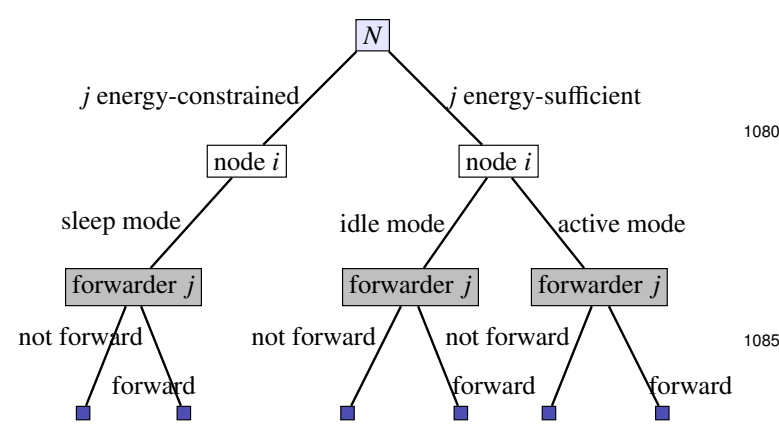

Figure 3: Extensive form of Bayesian game plays "not forward", when $B_{0}$ is low) is a pure BNE strategy. In contrast, when $B_{0}$ is high, a mixed strategy approach is presented to analyze BNE. The cooperation of the forwarder $j$ (i.e., playing "forward" or "not forward") is decided according to its payoff ${ }^{4095}$ Cooperation between the sensors can not be taken for granted, thereby cooperation enforcement represented by a credit-based incentive mechanism is defined in the game. A sensor will earn a reward $R$ if it forwards a packet for a neighboring sensor, where $R>0$. All $l_{100}$ the mathematical formulations of $j$ 's payoff in the the six different cases, illustrated in Fig. 33, are further described in [69]. Simulation results show that the proposal enhances the network lifetime, compared to the techniques such as Flood and AODV [70], by select $\overline{\overline{1}}_{105}$ ing the delivery path based on a sensor's energy. The proposed work has the lowest remaining energy distribution since it has the longest operation time due to the distribution of traffic loads/energy cost among different nodes.

\subsubsection{Discussion and Future Directions}

All the reviewed proposals in this domain are concentrated in some common issues. For example, in constructing or determining the energy aware paths, helping nodes in making decisions weather to take a part in routes or not (selfishness problem arises here) \$115 $_{315}$ or handling with nodes selfish behavior, in order to achieve a fair residual energy distribution which prolongs the network lifetime. The proposals differ from each others in the game class used, or the solution proposed. For instance, GTEBR [55] takes the residual ${ }_{120}$ energy of nodes as a main metric, while in [69], three factors are taken into account: energy, location, and cooperation between sensors. DSR-G [62] chooses reliable routes to prolong the network lifetime. The utility function of DSR-G considers four factors: collision $_{\$ 125}$ transmission power, reliability, and connectivity. In HDBR [64] the amount of data, bandwidth and delay are also considered as balancing criteria. In contrast, the energy in VGTR [57] is expressed using a time derivative the represents the amount of remaining life ${ }_{+130}$ time of a node on past workload is more informative. The reason is that the amount of energy alone does not always convey a practical meaning, as the value of energy is dependent on additional factors. In VGTR, the selection of a next hop is rotated to achieve energy balanced routing. It is worth mentioning that [69] considers WSNs' scenarios that allow mobility but it has a low remaining energy distribution.

In [69, 64], nodes depend on local information only, which reduces overhead. Both [68] and [69] use cooperation enforcement mechanisms to encourage a node's neighbor to forward its packets by employing a punishment and reputation-based scheme [68] or by giving rewards (credit-based system) [69]. It is worth noting that the usage of incentive mechanisms is very useful in this domain.

Computational intelligence is used in [67, 68] to reach a desired equilibrium with reduced complexity. Again, the usage of non-cooperative games outperforms other classes of games due to its simplicity and reduced overhead.

Received signal strength (link quality) is an important metric when identifying the best possible routes. Adding this metric to the cost function should be considered in the future design. Furthermore, although HDBR handles heterogeneous WSNs scenarios, the work in this direction still needs more efforts to study how the existence heterogeneous nodes affects the routing decisions and the overall performance of the WSN. Game theory has been also used to address energy savings in data aggregation based routing protocols which will be discussed later, in Subsection 5.1.

\subsection{Clustering}

Cluster formation is one of the early proposed methods for energy efficient operation in WSNs. It limits the scope of inter-clusters interactions to cluster heads and avoids redundant exchange of messages among nodes, while reducing the size of the routing table stored at each individual node. Cluster head selection process has a significant effect on the WSN performance. However, since it is a NP-hard problem, many heuristic methods, like LEACH, TEEN [71] and APTEEN [72], have been presented to solve it. They focus on balancing the energy consumption of nodes by dynamically changing cluster heads. However, these methods do not always guarantee an energy efficient operation during the whole lifetime of network. In fact, the most commonly used clustering schemes that are based on LEACH, are usually quite inefficient from network lifetime maximization point of view. This is because If any member inside a cluster dies, this typically should not affect the lifetime of the cluster, since it does not influence the transmission of other nodes [73]. Moreover, LEACH requires that all clusters perform direct transmission to the sink. Solutions proposed for multi-hop flat WSNs are not always feasible in cluster-based WSNs. 
Table 5: Proposals in WSN Clustering (Section 5.2)

\begin{tabular}{|c|c|c|c|c|c|}
\hline Article & Year & Algorithm & Game Class & Game Techniques & Method of Energy Savings \\
\hline [76] & 2012 & DEGRA & Non-cooperative & $\begin{array}{l}\text { NE, best response, perfect info, } \\
\text { pure strategy, dynamic game }\end{array}$ & $\begin{array}{l}\text { taking the residual energy and average energy con- } \\
\text { sumption of neighbors into consideration in the } \\
\text { cluster head selection process }\end{array}$ \\
\hline [77] & 2012 & GTC & Non-cooperative & win-stay, lose-shift & an energy efficient cluster size determination \\
\hline [79] & 2012 & - & Non-cooperative & $\mathrm{NE}$ & $\begin{array}{l}\text { a fair transmission load assignment between cluster } \\
\text { members }\end{array}$ \\
\hline [80] & 2010 & - & Cooperative & $\begin{array}{l}\text { coalition, Shapley value, cost- } \\
\text { sharing game }\end{array}$ & $\begin{array}{l}\text { the residual energy and the transmission distance } \\
\text { are taken into consideration when forming coali- } \\
\text { tions }\end{array}$ \\
\hline [73] & 2012 & - & Cooperative & NBS & $\begin{array}{l}\text { a fair resource allocation between clusters by mod- } \\
\text { eling the utility of cluster members as their lifetime }\end{array}$ \\
\hline [81] & 2012 & CSGC & Cooperative & $\begin{array}{l}\text { coalition, Shapley value, cost } \\
\text { sharing game }\end{array}$ & $\begin{array}{l}\text { the } \mathrm{CHs} \text { share only the cost of common affairs } \\
\text { among } \mathrm{CHs} \text { in inter-cluster transmission }\end{array}$ \\
\hline [82] & 2013 & - & Cooperative & Coalitions, Shapley axioms & $\begin{array}{l}\text { optimizing the coalition formation under control- } \\
\text { lable QoS constraints, and exploiting the correlated } \\
\text { data }\end{array}$ \\
\hline [83] & 2011 & - & Cooperation Enforcement & Auction mechanism, incentive & $\begin{array}{l}\text { electing the node with the most energy remaining } \\
\text { as a } \mathrm{CH} \text {, and encouraging selfish nodes to behave } \\
\text { honestly by providing incentives }\end{array}$ \\
\hline [85] & 2013 & AASA & Cooperation Enforcement & Auction mechanism & $\begin{array}{l}\text { the } \mathrm{CH} \text { activates only the nodes in the predicted re- } \\
\text { gion of the target, while the rest of nodes remain in } \\
\text { sleeping mode, then an auction mechanism is used } \\
\text { in the election of } \mathrm{CH} \text { to reduce energy }\end{array}$ \\
\hline
\end{tabular}

Different Computational Intelligence (CI) ap+165 proaches have also been proposed for energy aware cluster-based WSNs. Most of these approaches has been classified and discussed in [53]. Energy Hole Avoidance problem is also a crucial routing and clustering problem [74, 75]. This problem appears when ${ }_{170}$ nodes closer to the sink have to transmit more packets those far from it, as it depletes their batteries first, hence leaving a hole near the sink and partitioning the whole network. Uneven clustering is one of the methods proposed for load balancing in order to avoid such a situation. In this method, a smaller cluster radius near sink and a larger cluster radius away from the sink are defined, respectively.

Game theory is a suitable mathematical tool for optimizing energy-efficient clustering approaches in $_{180}$ WSNs due to the various and distributed models that it offers (e.g., coalitional games). In the following subsections, we present and discuss the latest proposals that use game theory in this domain. The proposals are further summarized in Table 5 .

\subsubsection{Non-cooperative Games}

A Density-based Energy efficient Game-theoretic Routing Algorithm (DEGRA) is presented in [76] for solving the conflict between an individual node and the $\mathrm{d}^{190}$ entire network. The goal is to improve the cluster head selection process. DEGRA sets a utility function to determine the $\mathrm{CH}$ based on nodes' density. The proposal takes both the residual energy of a node and the average energy consumption of its neighbors into con ${ }^{195}$ sideration. The $\mathrm{CH}$ selection problem is regarded as a k-stage dynamic game. Every player knows the utili- ties and strategies available to other players and each chooses its strategy based on the observation of previous stages. Thus, the game is a finite complete and perfect information game. The game has a pure strategy NE (i.e., every player is playing a best response to the strategy choices of its opponents) for each stage. Besides, all stages constitute a subgame perfect NE of the dynamic game. Simulation results show that DEGRA consumes less energy than LEACH due to the effective determination and distribution of $\mathrm{CHs}$.

Game Theoretic Clustering (GTC) [77] is an energyaware distributed algorithm proposed to adaptively determine suitable cluster sizes by tuning the width of nodes' regions. GTC consists of two parts: the load balancing algorithm, called LBA, and a cluster formation using the Win-Stay, Lose-Shift (WSLS) strategy. WSLS is based on the principle that if the most recent payoff is high, the same choice will be repeated, otherwise the choice will be changed [78]. Using WSLS, nodes at different locations can adapt their transmission ranges for "cluster-formation-announcement" rather than the constant ranges fixed at the beginning. Regions closer to the sink have smaller width, thus $\mathrm{CHs}$ at different hop distances could achieve similar energy consumption levels. Simulation results show that the network lifetime is extended when WSLS is adopted. Those results are specially significant when the node density is high. This is because that the $\mathrm{CH}$ role can be rotated among more nodes. The weakness of GTC is that it assumes there is only one $\mathrm{CH}$ in each region which limits its applicability.

To achieve energy fairness, the transmission load should be distributed among sensors such that, regard- 
less of a sensor's working conditions, no sensor node should be unfairly overburdened. In [79], the transmission load assignment in WSNs is modeled as a game ${ }_{1245}$ This work focuses on a cluster-based and surveillanceoriented sensor network. In fact, one report from a sensor in the cluster is enough to trigger the response of the surveillance system, and the other nodes can conserve their energy by just keeping silent. The keyr250 motivation of this work is to determine which sensor is going to report to the sink. The problem can be more complicated when it considers the heterogeneity of sensors. NE of the energy balancing game is derived. The NE solution can also meet the QoS re+255 quirements.

\subsubsection{Cooperative Games}

To balance energy consumption of nodes and maximize the network lifetime, a cooperative game the ${ }_{\uparrow 260}$ oretic model for clustering algorithms is proposed in [80]. The selfish behavior of nodes in non-cooperative games expedites network partition and results in an unfair residual energy distribution in them. Thus, the algorithm poses conditions for forming coalitions, $\operatorname{con}_{\uparrow 265}$ sidering the residual energy, transmission distance, and the number of nodes in a cluster. The nodes have to trade-off both individual cost and network-wide cost. Therefore, a cost sharing game is considered. Shapley value is chosen as a solution that assigns a single cost $_{270}$ allocation to the cost sharing game. Compared with other algorithms, this clustering scheme prolongs the network lifetime, reduces transmission time, and regulates the area of clusters to achieve energy efficiency.

In [73], a fair resource management approach for ${ }_{275}$ WSNs with a clustering scheme based on a bargaining game is proposed. The proposal assumes that each clusters has the same number of cluster members. The NBS is applied by modeling the utility of cluster members as their lifetime. The lifetime of a cluster member ${ }_{280}$ $i$ allocated to a time slot $n$ to communicate with its $\mathrm{CH}$ at a transmission rate $r_{i}(n)$ can be derived as follows:

$$
\tau_{i}(n)=\frac{E_{i}}{p_{i}(n)}=\frac{\left|h_{i i}(n)\right|^{2}}{I_{i}(n)\left(2^{r_{i}(n) / W_{i}(n)}-1\right)}
$$

where $E$ is the total battery energy, $p_{i}(n)$ is the transmission power of cluster member $i, \tau_{i}(n)$ is the lifetime of a cluster member $i$, which depends on the power strategy of cluster members in a time slot $n, W_{i}(n)$ is the bandwidth, $h_{i i}(n)$ is the channel gain of the link from290 a cluster member $i$ to its $\mathrm{CH}$ which belongs to cluster

${ }_{1235} i$, and $I_{i}(n)$ is the total interference and noise power at the $\mathrm{CH}$, which belongs to cluster $i$ during slot $n$. It is proved that the set of the achievable utilities of NBS is a convex set. An optimal point can be found (i.e., the NBS is unique). The algorithm is centralized and it is 295 valid only for single-hop transmission schemes.

A Cost Sharing Game-based Clustering (CSGC) [81] is a cooperative game that is used to solve the $\mathrm{CH}$ selection process. The paper presents a bi-directional cooperative clustering model, where cluster members cooperate in inter-cluster and intra-cluster transmissions. Similar to [80], a cost sharing game-based $\mathrm{CH}$ selection scheme is proposed to achieve an efficient management of clusters. For the purpose of simplicity and reducing the burden on $\mathrm{CHs}$, the cost that $\mathrm{CHs}$ share does not contain data transmission cost. CHs share only the cost of common affairs among other CHs (i.e., broadcasting information, launching cluster, synchronization, among others). A fair cost allocation is obtained through the use of Shapley value. $\mathrm{CHs}$ that join cost sharing are robust in residual energy and position. Besides, in case of dynamic clustering, CSGC can adapt the $\mathrm{CH}$ selection process to the changing constraints like the node position and the residual energy. The results show that CSGC outperforms LEACH on network lifetime, transmission capacity, and energy efficiency.

In [82], a scheme that employs a small number of nodes with computing power and large batteries, called "representatives", to optimize the coalition formation under controllable QoS constraints is proposed. The representatives may act either as local sinks, or as coordinators of operations performed either by sensors or by coalitions. The number and density distribution of representatives critically affect the overall network design. The spatial correlation of the data gathered is also exploited to formulate a cooperation scheme that reduces drastically the number of transmissions to save energy. The WSN lifetime maximization problem is accordingly transformed into a coalition formation game of three phases initialization, optimization, and steady-state phase. In order to save energy during the initialization phase, nodes interact only with their neighbors. The optimization of the initial coalition formation must be accomplished by the representatives. The proposed coalition formation satisfies Shapley axioms, and fairness in each coalition is guaranteed. Nodes belong to different coalitions generally have different coalitional values. The performance of such an approach is examined and compared to other clustering schemes. This proposal prolongs the lifetime of WSNs. The lifetime could be further extended by increasing the number of representatives. However, as the number of representatives increases, over partitioning will occur more frequently, reducing the aggregate benefit from coalition formation.

\subsubsection{Cooperation Enforcement Games}

The $\mathrm{CH}$ selection process is based on electing the node with the highest remaining energy within a cluster. The problem appears when there exist selfish nodes which lie about their remaining energy to avoid being elected. A solution based on an auction mechanism is proposed in [83]. It works as follows: Firstly, the nodes with the highest remaining energy is al- 

creases. An auction mechanism is proposed in 85 for the cluster formation process in order to reduce energy. The $\mathrm{CH}$ predicts the next location of the target and activates nodes in the predicted region, while the rest of nodes remain in sleeping mode. In [83], the proposed mechanism encourages selfish nodes to behave honestly by providing incentives in the $\mathrm{CH}$ selection process. The algorithm proposed in [73] is centralized, and it is not recommended for multi-hop transmissions. The contribution of CSGC [81] is that it presents a bidirectional cooperative clustering model, where cluster members cooperate in inter-cluster and intra-cluster transmissions. Overall, we can clearly notice that the nature of cluster-based WSNs is more suitable to be solved by using cooperative games, due to the formulation of coalitions and clusters.

In the future, specifying which nodes should be placed in the same cluster is a problem that could be addressed using game theory. Moreover, energy hole avoidance problem has been covered only by [77] using a distributed non-cooperative game. Therefore, more efforts should be allocated to this important problem.

\section{Coverage and Topology Control}

\subsection{Coverage}

The coverage control problem is defined by answering a fundamental question: how well do the sensors observe the physical space. This problem has been previously formulated in several ways. The investigation of coverage problems in WSNs is conducted in [86, 87].

In general, there exists a strong relationship between coverage and lifetime in WSNs. Unfortunately, improving one of these metrics comes at the expense of the other. A strategy that is commonly employed to achieve these two conflicting goals is to schedule only few nodes to be awake at any given point of time. This way the network lifetime is extended without compromising the coverage requirements. Therefore, the key challenge here is to design those scheduling algorithms based only on local information, aiming to achieve near optimal performance.

The set $k$-cover algorithm is an energy efficient coverage solution, whose goal is to determine whether every point in the service area is covered by at least $k$ sensors subject to lifetime requirements. However, the existing set $k$-cover algorithms [88, 89, 90] are centralized, and can not adapt to large-scale sensor network applications. Moreover, in [89, 90] it is proved that this problem is an NP-complete problem. There are some heuristic algorithms in the literature to find the cover sets. For example, [91] proposes a polynomial-time algorithm in terms of the number of sensors. The algorithm can be easily transformed into distributed protocols. Worst and best case coverage are also a wellknown problem which is discussed in [86]. In [92], an efficient distributed algorithm to find an optimum best-coverage-path with the least energy consumption is presented. 
Table 6: Proposals in WSN Coverage (Subsection 6.1)

\begin{tabular}{|c|c|c|c|c|c|}
\hline Article & Year & Game Class & Distributed/Centralized & Game Techniques & Method of Energy Savings \\
\hline [93] & 2007 & Non-cooperative/Cooperative & Distributed & NE, regret strategy & $\begin{array}{l}\text { maximizing the area coverage subject } \\
\text { to a lifetime guarantee }\end{array}$ \\
\hline [95] & 2008 & Non-cooperative & Distributed & $\mathrm{NE}$ & $\begin{array}{l}\text { maximizing the area coverage subject } \\
\text { to a lifetime guarantee }\end{array}$ \\
\hline [96] & 2009 & Non-cooperative & Distributed & $\mathrm{NE}$, repeated game & $\begin{array}{l}\text { maximizing the area coverage subject } \\
\text { to a lifetime guarantee }\end{array}$ \\
\hline [97] & 2008 & Non-cooperative/Cooperative & Centralized & NE, repeated game & $\begin{array}{l}\text { adjusting the coverage range of nodes } \\
\text { while controlling the transmission } \\
\text { power }\end{array}$ \\
\hline [98] & 2009 & Non-cooperative & Distributed & repeated game & $\begin{array}{l}\text { optimizing the transmission range dy- } \\
\text { namically in order to save energy }\end{array}$ \\
\hline$[100]$ & 2013 & Non-cooperative & Distributed & $\begin{array}{l}\text { best response, exact poten- } \\
\text { tial game, repeated game }\end{array}$ & $\begin{array}{l}\text { optimizing the area coverage while } \\
\text { minimizing energy costs }\end{array}$ \\
\hline [104] & 2010 & Cooperative & Distributed & $\begin{array}{l}\text { Kalai-Smordinsky bargain- } \\
\text { ing solution }\end{array}$ & $\begin{array}{l}\text { a compromise model for both energy } \\
\text { conservation and sensing accuracy }\end{array}$ \\
\hline [105] & 2013 & Cooperation Enforcement & Distributed & $\begin{array}{l}\text { repeated game, incomplete } \\
\text { info, incentive mechanism } \\
\text { (reputation-based) }\end{array}$ & $\begin{array}{l}\text { optimizing the network coverage tak- } \\
\text { ing the energy efficiency and the selfish } \\
\text { behavior into account }\end{array}$ \\
\hline
\end{tabular}

\subsubsection{Non-cooperative Games}

In [93], the Distributed, Robust and Asynchronous Coverage (DRACo) algorithm is presented. Its goal is to solve the set $k$-cover problem in order to provide the maximum possible coverage subject to lifetime constraints. It is assumed that $N=|\mathcal{N}|$ nodes are randomly scattered in a field of area $A$ with sensing and communicating range $R_{S}$ and $R_{c}$, respectively. Every node belongs to one of $k$ disjoint sets. Time is slotted and most of energy is consumed during the active slots. Nodes are scheduled to be active over a schedule of length $k$, such that in each slot $i$, nodes belonging to set $i$ are active. Therefore, the lifetime of the network is proportional to $k$. Given such a schedule, the objective is to determine the optimal $k$ partition of $\mathcal{N}$, such that the average coverage is maximized. The optimization problem is formed as follows:

$$
\begin{gathered}
\max C(s)=\frac{1}{k} \sum_{i=1}^{N} C_{i}(s) \\
\text { s.t. } s \in \mathcal{S}
\end{gathered}
$$

where $\mathcal{S}=\left\{s_{1}, s_{2}, \ldots s_{N}\right\}$ represents a partition of $\mathcal{N}$ into $k$ cover sets (i.e., $\mathcal{S}$ is the set of all possible $k$ covers). $C(s)$ is the coverage metric which depends on the topology of the network, the sensing range $R_{s}$ of the nodes, and on $k$. A key challenge is to achieve this 445 partition in a distributed manner with purely local information and yet provide near optimal coverage. For a node $i$, which has chosen a slot $s_{i}$, if all of its sensing region is covered by other, then node $i$ is redundant

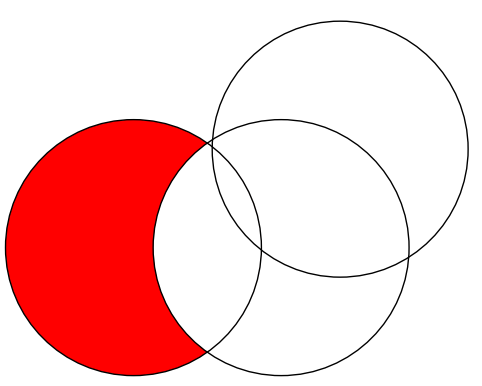

Figure 4: The payoff of node $i$ is the red area

in slot $i$. Therefore, if node $i$ switches to a slot where there is region covered only by node $i$, the coverage performance would be improved (see Fig. 4). The concept called "the regret of a node", defined in graphical games [94], is used. The game converges to NE in a purely distributed way. Moreover, DRACo is robust to network dynamics and can converge even when executed asynchronously. The simulations indicate that the convergence speed of DRACo is almost constant with the number of nodes $N$ and $k$.

The work done in [95] is an extension of [93]. It proposes synchronous and asynchronous algorithms, which converge to pure NE. Moreover, it analyzes the optimality and complexity of pure NE in the coverage game via the price of anarchy [3]. It is proved that, the ratio between the optimal coverage and the worst case $\mathrm{NE}$ coverage, is upper bounded and depends on the maximum number of nodes which cover any point in the NE solution. It is also proved that finding the pure $\mathrm{NE}$ in the general coverage game is PLS-complete. Extensive simulations show that, the NE coverage performance is very close to the optimal coverage and the convergence speed is sub-linear. Even under the noisy environment, the algorithms can still converge to the NE point. 
In [96], [93] is further extended, addressing the same problem by proposing a distributed algorithm. In [95], the maximum coverage set algorithm is proposed and [96] utilizes the maximum coverage set as the upper limit of the coverage set division. On the basis of this lapping Subfields (MLOF), satisfying division condition, as node's utility function. Then, it puts forward a distributed heuristic algorithm to get optimal strategy by iteration in order to reach NE. Using game theory, network lifetime is maximized while ensuring the maximum area coverage. However, if the destiny of nodes is high, increasing the node coverage set will ${ }^{485}$ lead to an increase of algorithm iteration number.

In [97], Game-theoretical Complete Coverage (GCC) is proposed to ensure complete network coverage through adjusting the coverage range of nodes and controlling the redundancy in network coverage ${ }^{1490}$ This paper takes into account transmission power control. The problem description is that in a network that has changed its topology due to mobile or sleeping nodes, parts of nodes' coverage area should be decided again. In this case, every node probably has to update its operating parameters. In many cases, the way to solve this problem is through a detailed planning of the network topology by optimizing the coverage area of every node. This approach is easy to manage but not suitable for the stability of a WSN whose topology changes fast. The aim of GCC is to avoid the series of holes in coverage rapidly and effectively. Game theory is used to optimize this problem and the payoff of every node is defined as follows:

$$
\text { Payoff }=1-\alpha r_{i}^{2}+\beta p_{i}
$$

where the value of the complete coverage is $1, r_{i}$ is the ranges between 0 and 1 . It affects the amount of energy consumption. Smaller coverage area saves more energy. $\beta$ is a parameter (between 0 and 1) related to the importance of the coverage level that should be considered during the nodes' decision, and $p_{i}$ is the coverage level. The strategy of power management is that in each stage of game, the sink broadcasts energy level data. Then, an energy level determined through the $\mathrm{NE}$ confirmed. If any node deviates, other nodes ${ }^{495}$ will increase their energy levels in order to punish it. All the nodes know other nodes energy condition. In every stage of game, the energy distribution of every node achieves equilibrium. During the whole repeated game processes, certain equilibrium is reached, which is more effective than single-stage NE. AN improve +500 ments over GCC is proposed. The two versions -the basic and the improved- are compared. Nevertheless, this proposal is not fully distributed since the sink plays an essential role.

Game-theoretical Total Link (GTL) [98] is an algo+505 rithm based on game theory designed for optimizing the transmission range dynamically in order to save energy. An amount of $20 \%$ of energy is saved in comparison to the Critical Transmitting Range (CTR) algorithm [99], where sensors assign fixed and equal transmission ranges, which results in coverage overlapping (i.e., energy waste). Nodes control their energy consumption flexibly according to topology changes without loss of connectivity and robustness. For doing so, the following payoff function of a node is proposed:

$$
\pi_{i}=1-\alpha r+\beta(n-k)
$$

where $r$ is the transmission range radius, $\alpha$ and $\beta$ are weight parameters, $n$ is the amount of neighbors, and $k$ is the neighbor expectation. $\beta(n-k)$ is the benefit from neighbors, and $-\alpha r$ represents the energy consumption. Through repeated games, the whole network will reach a NE which means that most nodes will decrease their energy consumption. The paper shows that in experiments with a sleeping strategy, the sleeping nodes would probably make extra energy waste than other active nodes.

The work presented in [100] formulates the coverage optimization problem for mobile sensors as a constrained repeated multi-player strategic game. Each sensor tries to optimize its own coverage while minimizing the processing energy cost. A number of learning rules (e.g., best response dynamics and adaptive play) have been proposed to reach NE. Utility values induced by alternative actions are inaccessible because of the information constraints caused by unknown rewards, motion, and sensing limitations. To tackle this challenge, two distributed payoff-based learning algorithms are developed, where each sensor remembers only its own utility values and actions played during the last two rounds. These algorithms are proven to be convergent to the set of constrained NE and global optimum of a certain coverage performance metric. The utility function proposed for an agent $i$ that aims to capture the sensing/processing trade-off is:

$$
u_{i}(s)=\sum_{q \in D\left(a_{i}, c_{i}\right) \cap Q} \frac{W_{q}}{n_{q}(s)}-f_{i}\left(c_{i}\right)
$$

The first term of the formula represents the benefit that agent $i$ obtains through sensing. The second term represents the sensing energy/processing cost. This coverage game is proved to be a constrained EPG [101]. More results of this work are presented in [102, 103].

\subsubsection{Cooperative Games}

In [104], a compromise model based on a cooperative game for both energy conservation and sensing accuracy is proposed. A realistic sensing model which allows high flexibility in optimizing networks' sensing activity is presented. The interaction between sensor nodes is modeled as a cooperative bargaining 
game, where individual sensors cooperate for achieving the application sensing requirements while minimizing and balancing the energy consumption. Kalai-

1510 Smordinsky Bargaining Solution (KSBS) is used to find a distribution rule that optimizes the trade-off in ${ }_{565}$ the compromise problem. Simulation results show that the WSN lifetime is extended, compared to a Linear Programming $[\mathrm{LP}]$ and a heuristic methods.

\subsubsection{Cooperation Enforcement Games}

In [105], a Coverage Maintenance Protocol (CMP) that is based on game theory is presented. An incentive mechanism is used to tackle the selfish behavior of nodes. Selfish nodes may refuse to wakeup to exthe eligibility of a sensor node to sleep) for one or several rounds, in order to save more energy and increase their lifetime. The objectives of this work are to detect and prevent such behavior, and to optimize the network corage. In addition, CMP assures that the network coverage degree is maintained by the remaining active nodes. Thus, it helps to balance the energy consumption by scheduling the active state of nodes. The game is considered a multi-stage repeated game, since the

1530 phases of coverage optimization and maintenance con- ${ }^{155}$ sists of several rounds. The sent and received control packets overhead is evaluated according to the number of detected selfish nodes. Results show that the energy efficiency and the network lifetime are affected when the number of selfish nodes increases. The results reveal a trade-off between coverage range (i.e., accuracy of selfish node detection) and the control packet overhead, as it affects energy efficiency.

\subsubsection{Discussion and Future Directions}

Designing a distributed energy-saving solution in this domain is a challenge that has attracted such attention. All the previous set $k$-cover algorithms are centralized. Besides, it can not adapt to large-scale sensor network applications. In [93, 95, 96], game the ${ }_{+600}$ ory has been applied to address the $k$-cover problem in order to provide the maximum possible coverage subject to a lifetime guarantee. However, the algorithm in [93, 95] has many limitations as discussed in [96]. Firstly, the network lifetime is related to the number 605 of coverage node sets. Secondly, the algorithm aims at maximizing lifetime at the expense of overall coverage. This is against the user demands in real monitoring applications, when it is required to enlarge the coverage area as far as possible. Thirdly, the algorithm ${ }_{610}$ can not achieve a balance between network node density and the number of coverage sets number to optimize the network coverage area. Lastly, this algorithm uses node's exposed area as payment function, which makes calculations in the real application complicated ${ }_{615}$ and less accurate. In [96], if node destiny is high, in- creasing the node coverage set number will lead to an increase of algorithm iterations.

Coverage control for mobile WSNs is addressed in GCC [97] and [100]. GCC allows nodes to adjust their coverage range by taking transmission power control into account. The main drawbacks of GCC are that all nodes should know other nodes' energy levels. Moreover, the sink plays an essential role by broadcasting energy level data. In contrast, in [100] the utility values induced by alternative actions are inaccessible because of the information constraints. Besides, it employs an accurate sensing model. GTL[98] optimizes transmission ranges dynamically to reduce coverage overlaps in order to save energy.

On the other side, cooperative games are not widely used in this domain. Only one proposal in the recent literature uses a cooperative game to address the trade-off problem between sensing accuracy and lifetime by using scheduling techniques -not a pure coverage problem- in [104]. Finally, cooperation enforcement games are useful when some nodes might have a selfish behavior or deviate from the NE point (see [105]).

In the future, the collaborative relation between the coverage control and the MAC layer (e.g., scheduling nodes to sleep according to the required coverage) will be an important issue that could be modeled using game theory tools. Moreover, game theory could also be used to address the joint coverage and power control problems. For example, the presence of multiple wireless networks in ISM bands, including Wireless Local Area Networks (WLANs) and other WSNs, is a cause of mutual interference. WSNs may use dynamic channel hoping to avoid interference from external networks by moving all or one part of the network to a different channel [106]. To do that, all sensors should agree on that decision as the network connectivity must be guaranteed.

\subsection{Topology Control}

In some applications, nodes need to be placed accurately at predetermined locations. Given a geographical coverage, Topology Control (TC) determines where to place nodes, cluster heads (in cluster-based topologies), or sinks. It helps in arranging the communication among them.

The network lifetime during which the topology is preserved -or adapts dynamically- is referred to as topological lifetime. Many researchers aimed to maximize this topological lifetime with regard to a given mission and a certain amount of initial energy. One energy savings strategy is to allow each node to adjust its transmission power to cover only a specific set of direct neighbors, while preserving connectivity and coverage. A survey on distributed TC techniques for prolonging the lifetime of WSNs is provided in [107]. However, the failure of nodes due to energy depletion 
Table 7: Proposals in WSN Topology Control (Subsection 6.2)

\begin{tabular}{|c|c|c|c|c|c|c|}
\hline Article & Year & Algorithm & Game Class & Distributed/Centralized & Game Techniques & Method of Energy Savings \\
\hline [109] & 2008 & DIA & Non-cooperative & Centralized & $\begin{array}{l}\text { potential game, Pareto effi- } \\
\text { cient }\end{array}$ & $\begin{array}{l}\text { preserving network connectivity with a fair } \\
\text { transmission power distribution }\end{array}$ \\
\hline [112] & 2009 & EPG,OPG & Non-cooperative & Distributed & $\begin{array}{l}\text { exact and ordinal potential } \\
\text { game }\end{array}$ & $\begin{array}{l}\text { preserving network connectivity with a fair } \\
\text { transmission power distribution }\end{array}$ \\
\hline [113] & 2009 & $\begin{array}{l}\text { Joint } \\
\text { TC and } \\
\text { Power }\end{array}$ & Non-cooperative & Distributed & $\begin{array}{l}\text { dynamic game, sequential } \\
\text { move, static game, BNE, } \\
\text { incomplete info }\end{array}$ & $\begin{array}{l}\text { the transmission power control is consid- } \\
\text { ered in the TC procedure }\end{array}$ \\
\hline [114] & 2012 & VGEB & Non-cooperative & Distributed & $\begin{array}{l}\text { potential game, incomplete } \\
\text { info, Pareto efficient }\end{array}$ & $\begin{array}{l}\text { reducing the energy waste when exchang- } \\
\text { ing the information, and selecting the nodes } \\
\text { with higher energy as direct neighbors }\end{array}$ \\
\hline [115] & 2012 & NS & $\begin{array}{l}\text { Non- } \\
\text { cooperative/Cooperative }\end{array}$ & Distributed/Centralized & $\mathrm{NE}$, best-response & $\begin{array}{l}\text { optimizing the energy consumption by con- } \\
\text { necting with a minimal set of neighbors and } \\
\text { using the minimal transmission power }\end{array}$ \\
\hline [116] & 2012 & CTCA & Non-cooperative & Distributed & $\mathrm{NE}$, ordinal potential game & $\begin{array}{l}\text { a node makes a sacrifice by increasing its } \\
\text { transmission power dynamically if it can } \\
\text { help their neighbors (with short lifetime) to } \\
\text { reduce energy consumption }\end{array}$ \\
\hline [117] & 2014 & - & Non-cooperative & Distributed & BNE & $\begin{array}{l}\text { efficient placement of relay nodes to } \\
\text { guarantee network recovery in partitioned } \\
\text { WSNs }\end{array}$ \\
\hline [118] & 2010 & - & Cooperative & Distributed & $\begin{array}{l}\text { coalition, } \\
\text { superadditive, rewards }\end{array}$ & maximizing the feasible sleep time \\
\hline
\end{tabular}

may partition the network leaving some areas uncovered. Moreover, it has a negative effect in the application since it prevents data exchange. Therefore, topology management techniques for tolerating node failures in WSNs have been surveyed in [108].

Since topology may vary with time due to malfunctioning nodes or node mobility, it is preferable that the network is able to dynamically adjust the topology in a distributed manner. The TC algorithms found in the literature are either centralized algorithms (i.e., require global network information), semi-distributed or distributed algorithms [107, 109]. However, a central coordination is often impractical, therefore distributed approaches are required. In this context, a simple distributed topology control algorithm that determines the minimal power consumption operating point for each node in a multi-hop wireless ad hoc network is proposed in [110]. There each node makes local decisions about its transmission power. These local decisions collectively guarantee global connectivity.

Game theory models can effectively address the process of nodes' deployment and control their transmission power in order to reach a solution which optimizes energy efficiency and prolongs the network lifetime ${ }_{i 66}$ The following subsections will discuss the latest proposals in this domain. The papers are summarized in Table 7

\subsubsection{Non-cooperative Games}

Delta-Improvement Algorithm (DIA) [109] is a TC game that extends the Max-Improvement Algorithm (MIA) [111]. The utility function $u_{i}$ of the game specifies that nodes have enough incentives to establish and maintain connectivity with a sufficient number of 675 neighbors, and ensures that the network does not parti- tion. It can be expressed as follows:

$$
u_{i}(p)=\varphi_{i}(g(p))-X_{i}\left(p_{i}\right)
$$

where $\varphi_{i}$ represents the benefit (i.e., being connected) node $i$ derives from network $g$, and $X_{i}$ is the cost (i.e., energy). This TC game is a potential game which guarantees the existence of a NE. The game also admits many locally efficient NE. However, only a subset of those NE topologies is globally efficient from an energy efficiency point of view. The problem with MIA is that, although it converges to topologies that preserve network connectivity, being greedy leads to a biased steady-state power-level distribution. In DIA, each node makes small decrements in its power level if that change improves its utility. Otherwise, the node reverts to its previous power level. Thus, at the end, the transmission power distribution is more fair. This work shows that under DIA, the induced topologies are energy efficient and preserve network connectivity. It is observed that the NE topology obtained by DIA is Pareto efficient. For any random topology, and from the Pareto efficiency and uniqueness of NE, it can be deduced that the steady-state power allocation under DIA is lifetime optimal.

In [109], an algorithm that guarantees convergence to a connected network is proposed. The algorithm requires global information flowing through the network in order to check at each iteration the connectivity of the network. [112] relaxes that assumption and proposes a fully distributed algorithm based on local information only to adjust the transmission power of each node in a WSN. Hence, the network becomes connected with an energy efficient solution. The algorithm is formulated as a non-cooperative game where nodes exchange information only with their neighbors 
(i.e., local information only). Potential games concepts (i.e., EPG and OPG) are used to prove the existence of NE. The results indicate that for a relatively low node density, the probability that the proposed algorithm leads to a connected network is close to one.

Power management and TC are directly correlated. The work presented in [113] is motivated by this consideration. It proposes a joint topology and power conrol algorithm based on game theory to analyze the decentralized interactions among heterogeneous sensors. Three desirable characteristics: reliability, connectivity, and power efficiency, are considered in designing the game. The strategies played by the nodes reflect the trade-off between Frame Access Rate (FSR), node degree, and power consumption. The power control problem is formulated into a realistic incomplete information dynamic game model with sequential moves. Two solution schemes for implementations are provided, NEPow and BEPow. The NEPow scheme is derived from the NE of the static game model. The BEPow scheme is derived from the BNE of the incomplete information dynamic game model. Both NE and BNE are proved under sufficient conditions. Simulations are done to examine the efficiency and stability of the proposed approach. The results show that the average transmission power over all nodes is reduced by $45 \%$ compared with the case without power control.

In many game theory based TC algorithms, every node has to make others aware of its actions by transmitting the control information repeatedly. This results in much unnecessary energy waste and network lifetime minimization. To solve this problem, a distributed Virtual Game-based Energy Balanced TC algorithm (VGEB) with incomplete information is proposed in [114]. In VGEB, each node only needs in ${ }^{473}$ formation exchange just once. Then, based on the obtained information, each node can figure out its own transmission power by executing a virtual game. This work illustrates that the TC virtual game is a potential game and can converge to the NE, which is Pareto ${ }^{74}$ optimal. Moreover, VGEB can easily construct the topology with a low information complexity of $O(n)$, and the induced topology can maintain the network connectivity, where $n$ is the number of nodes in network. VGEB is also compared with DIA. Simulation 745 results show that VGEB outperforms DIA in: i) balancing nodes' energy consumption by selecting some of the available nodes with higher energy as their dineighbors, ii) reducing the energy waste in information exchange, and iii) prolonging the lifetime of 750 network. In addition, the average-hops and maximumhops of the shortest path between a node pair in VGEB are much shorter than that in DIA. Hence, VGEB reduces the end-to-end delay.

The Neighbor Selection (NS) game is presented in 755 [115]. In this game, each individual node tries to selfishly choose its neighborhood such that its own en-

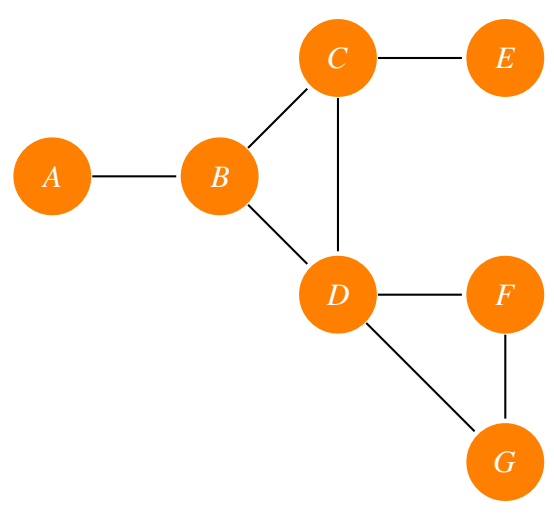

Figure 5: A sample NE topology

ergy consumption is optimized. The goal of nodes in this game is different in the sense that each node tries to egoistically optimize its energy consumption. This goal is achieved through connecting themselves to a minimal set of neighbors while also using the minimal transmission power. The choice of a minimal neighbor set allows nodes to minimize their traffic load. This objective creates a new game with completely different outcomes than the original TC game, where nodes are only interested in minimizing their transmission power. The utility function of node $i$ can be expressed as:

$$
u_{i}(L)=M f_{i}-\sum_{j,(i, j) \in E^{L}} v_{i, j} p_{i}
$$

where $f_{i}$ is the number of nodes connected to node $i$, $M$ is a fixed benefit multiplier. The negative term represents the energy cost, where $v_{i, j}$ is the volume of traffic going over the link $(i, j)$, and $p_{i}$ is the transmission power of node $i$ to any of its neighbors. The multiplier $M$ is set to a value larger than any possible energy cost value. The benefit term indicates that nodes prefer connectivity over energy savings. However, they would get more rewards by maintaining this connectivity with a lower energy usage. Hence, a connected topology is always preferred by nodes over a disconnected one.

Fig. 5illustrates a sample NE topology in which no node benefits from removing any of its non-cut links. In [115], a simplified version of this game where nodes know their transmission power before participating in the game is proposed first. Then, a couple of distributed algorithms is proposed to obtain stable topologies in a network of selfish nodes using both global and local connectivity information. The general case where the transmission powers are unknown variables is taken into consideration. The results show that the global method yields to about $20 \%$ higher total energy consumption than the approximated (stable) solution. However, if the local information is appropriately chosen, the local method can reduce this gap by more than $10 \%$.

Cooperative TC with Adaptation (CTCA) [116] is a 
dynamic TC algorithm based on game theory that considers both energy costs across links and the amount of maximizing the network's lifetime into an ordinal potential game. This allows a node running CTCA to make a sacrifice by increasing its transmission power dynamically if it helps in reducing the energy consumption at another node that has a shorter lifetime (see Fig. 6). The existence of NE is proved. Simulation results indicate that CTCA extends the life of a network by more than $50 \%$ compared to well-known algorithms.

Placing relay nodes is a possible solution to restore connectivity in partitioned WSNs. However, the existing solutions require some global information, regarding the availability of the number of partitions, and the location of the remaining nodes, among others, which may not be available in all applications. A distributed game theory based approach for the placement problem of relay nodes is proposed in [117], in order to guarantee network recovery for partitioned WSNs. Movement decisions of the relays are regarded as a net- ${ }^{1815}$ work game. A BNE function is assigned to each partition using limited information about the routes and partition boundary nodes. A probability distribution function is defined for each partition using this estimated equilibrium function (i.e., BNE). This game al- ${ }^{182}$ lows some relay nodes (the leaders) to determine the partition to be connected with (i.e., for recovery) based on the probability distribution function of the partitions. The recovery process proceeds with the partition with the next highest priority until the network is com- ${ }^{-182}$ pletely recovered (i.e., reaching the system-wide NE). Results show that this approach performs slightly better than a centralized approach in terms of the distance traveled by all relay nodes between partitions, which enhances the network lifetime. However, taking the ${ }^{1830}$ residual energy of the nodes into account when making decisions is planed for the future work.

\subsubsection{Cooperative Games}

Given that target localization requires nodes cooper- ${ }^{183}$ ation, the main idea of [118] is to dynamically achieve an optimal formation of collaborative coalitions. For this reason, a non-superadditive cooperative game is proposed. The term non-superadditive means that the grand coalition (i.e, the coalition comprising all nodes) is not optimal. Nodes in each coalition share measurements to localize a particular target. As a result, they are rewarded with sleep times. The paper explains why the optimal coalition does not necessarily comprise the nearest nodes to the target. In general, finding the optimal coalition structure is an NP-complete problem.

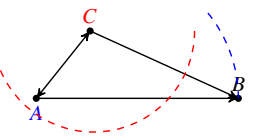

(a) The initial topology where no node can reduce its transmission power

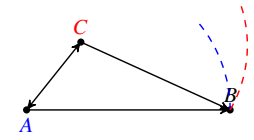

(b) Node C sacrifices and increases its transmission power to directly connect to $\mathrm{B}$

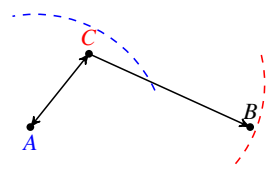

(c) Node A can now reduce its transmission power and connects only with $\mathrm{C}$

Figure 6: An example illustrating cooperative topology control

\subsubsection{Discussion and Future Directions}

A reader can clearly see the strong relation between topology and coverage problems (i.e., adjusting transmission range control and scheduling which nodes must turn on/off and when). When designing an energy efficient TC solution it is also crucial to maintain connectivity and fast convergence to a $\mathrm{NE}$ point. Proposals like [109, 112, 115, 117] use noncooperative games to achieve this problem. Potential games are also appropriate to solve this kind of problems (see [109, 112, 114, 116]). They are easy to implement and guarantee the convergence to NE. In addition, [114, 115] reduce the unnecessary energy waste in information exchange. CTCA [116] considers both energy costs across links and the amount of energy available on a node. A node running CTCA makes a sacrifice by increasing its transmission power dynamically if it helps in reducing the energy consumption of another node which has a shorter lifetime.

Given that the target localization requires nodes cooperation, a coalitional game is proposed in [118], which is the only cooperative game found in the literature in this domain.

To the best of our knowledge, the use of game theory in this domain usually assumes homogeneous wireless nodes. In the future, addressing the connectivity and bi-directionality issues in heterogeneous WSNs should be studied (e.g., nodes with higher hardware capabilities can help others to execute their tasks efficiently).

\section{Data Aggregation, Security, Task Allocation and Energy Harvesting}

\subsection{Data Aggregation}

Transmitting all sensor data, specially in dense WSNs, can result in a high traffic load and cause congestion at destination nodes. This may result in higher 
Table 8: Proposals in WSN Data Aggregation, Security, and Task Allocation (Section 7)

\begin{tabular}{|c|c|c|c|c|c|c|}
\hline Domain & Article & Algorithm & Year & Game Class & Game Techniques & Method of Energy Savings \\
\hline \multirow[t]{2}{*}{ Data aggregation } & [119] & CAR & 2012 & $\begin{array}{l}\text { Non- } \\
\text { cooperative }\end{array}$ & exact potential game & $\begin{array}{l}\text { the cost function takes into account the } \\
\text { energy and the correlated data }\end{array}$ \\
\hline & [125] & RGTAGN- ACO-R & 2012 & $\begin{array}{l}\text { Non- } \\
\text { cooperative }\end{array}$ & $\begin{array}{l}\text { reverse game, repeated game, } \\
\text { pure strategy }\end{array}$ & $\begin{array}{l}\text { an energy aware data aggregation by a } \\
\text { robust selection of aggregation nodes }\end{array}$ \\
\hline \multirow[t]{6}{*}{ Security } & {$[136]$} & - & 2014 & $\begin{array}{l}\text { Non- } \\
\text { cooperative }\end{array}$ & $\begin{array}{l}\text { evolutionary game, incomplete } \\
\text { info, mixed strategy }\end{array}$ & $\begin{array}{l}\text { finding the best strategy that balances } \\
\text { between the rewards and the costs re- } \\
\text { sulted from deploying the security mea- } \\
\text { sures (i.e., energy consumption) }\end{array}$ \\
\hline & [138] & TDDG & 2014 & $\begin{array}{l}\text { Non- } \\
\text { cooperative }\end{array}$ & mixed strategy NE & $\begin{array}{l}\text { an energy aware trust derivation } \\
\text { dilemma game }\end{array}$ \\
\hline & [139] & - & 2014 & $\begin{array}{l}\text { Non- } \\
\text { cooperative }\end{array}$ & $\begin{array}{l}\text { evolutionary game, mixed strat- } \\
\text { egy NE, best response }\end{array}$ & $\begin{array}{l}\text { an energy aware coordinator selection } \\
\text { mechanism }\end{array}$ \\
\hline & {$[140]$} & TEER & 2009 & $\begin{array}{l}\text { Cooperation } \\
\text { Enforcement }\end{array}$ & $\begin{array}{l}\mathrm{NE}, \text { incentive mechanism } \\
\text { (reputation-based) }\end{array}$ & $\begin{array}{l}\text { electing healthy } \mathrm{CHs} \text { with the highest } \\
\text { energy and trust levels }\end{array}$ \\
\hline & [141] & - & 2013 & $\begin{array}{l}\text { Cooperation } \\
\text { Enforcement }\end{array}$ & $\begin{array}{lr}\text { incentive } & \text { mechanism } \\
\text { (reputation-based), } & \text { central- } \\
\text { ized } & \end{array}$ & $\begin{array}{l}\text { maintaining a good reputation while } \\
\text { saving energy resources }\end{array}$ \\
\hline & [142] & G-FQL & 2013 & $\begin{array}{l}\text { Cooperation } \\
\text { Enforcement }\end{array}$ & $\begin{array}{l}\text { incentive mechanism (credit- } \\
\text { based), centralized }\end{array}$ & $\begin{array}{l}\text { to defend against attackers who send } \\
\text { flooding messages that exhaust nodes' } \\
\text { energy and prevent nodes from entering } \\
\text { the sleeping mode }\end{array}$ \\
\hline \multirow[t]{4}{*}{ Task allocation } & [153] & NGTSA & 2011 & $\begin{array}{l}\text { Cooperation } \\
\text { Enforcement }\end{array}$ & $\begin{array}{l}\text { mechanism design, incentive } \\
\text { mechanism (credit-based), pri- } \\
\text { vate information }\end{array}$ & $\begin{array}{l}\text { splitting the main tasks received by sink } \\
\text { into a number of sub-tasks and dis- } \\
\text { tributing them to the clusters }\end{array}$ \\
\hline & [154] & Centralized WDP & 2011 & $\begin{array}{l}\text { Cooperation } \\
\text { Enforcement }\end{array}$ & $\begin{array}{l}\text { reverse auction game, incom- } \\
\text { plete information }\end{array}$ & $\begin{array}{l}\text { maximizing the network lifetime by } \\
\text { sharing the tasks and the network re- } \\
\text { sources among applications }\end{array}$ \\
\hline & {$[156]$} & Distributed ED-WDP & 2012 & $\begin{array}{l}\text { Cooperation } \\
\text { Enforcement }\end{array}$ & $\begin{array}{l}\text { reverse auction game, incom- } \\
\text { plete information }\end{array}$ & $\begin{array}{l}\text { an energy and delay efficient decentral- } \\
\text { ized WDP mechanism }\end{array}$ \\
\hline & {$[157]$} & - & 2012 & $\begin{array}{l}\text { Cooperation } \\
\text { Enforcement }\end{array}$ & $\begin{array}{l}\text { incentive mechanism, mixed } \\
\text { strategy, repeated game }\end{array}$ & $\begin{array}{l}\text { serving nodes with the lowest remain- } \\
\text { ing energy level first }\end{array}$ \\
\hline
\end{tabular}

energy consumption for the overall network. A multihop WSN can reduce network traffic by aggregating data along the route from the nodes to the sink (see Fig. 7). This is achieved by using functions such as suppression (i.e., eliminating duplicates), min, $\max { }^{188}$ and average. Most routing algorithms in WSNs aim to minimize the total transmission cost of transporting the collected data in a distributed manner. Taking into account data correlation, as well as transmission energy per bit in routing decisions, we can improve the ${ }^{1885}$ system performance [119].

Data aggregation and in-network processing techniques depend on the type of data used in each specific application and must be tailored to it. A survey of tra ${ }_{\top 890}$ ditional data aggregation algorithms used in WSNs is presented in [120]. This technique has been used to achieve energy efficiency and traffic optimization in a number of routing protocols [58, 121, 122]. In [123], a structure-free data aggregation protocol (i.e., no using 895 any structure like tree-based or cluster-based) is proposed in order to reduce the delay and the energy spent on building and maintaining the data aggregation structure, specially in those environments where nodes may move or fail.

Game theory models are used to achieve an energy efficient data aggregation in a way that does not affect the network lifetime. The proposals are discussed below and summarized in Table 8

\subsubsection{Non-cooperative Games}

Correlation Aware Routing (CAR) [119] is an adaptive and distributed routing algorithm based on potential games. It is proposed to address the problem of designing an energy efficient transmission structure in WSNs where all nodes aggregate correlated data over intermediate nodes on a route to the sink. The total amount of energy consumed to correctly deliver one data symbol, accounting for data redundancy through correlation is calculated. The cost function takes into account the energy consumption, the interference, and correlated data. CAR is proved to be an EPG, for which a best response strategy is shown to converge to a NE. The performance of CAR is compared with Minimum Energy Routing (MER) schemes and MEGA [124]. Simulation results show that CAR outperforms both algorithms in saving the total effective energy in normal and dense networks. However, the end-to-end transmission delay minimization is not taken into consideration in this work.

Reverse Game Theory based Aggregator Node Selection and Ant Colony Optimization based Routing (RGTAGN-ACO-R) [125] is a novel framework for power efficient data aggregation in WSNs. The goal is to maximize the lifetime of the sensor network. The proposed system has two phases. In the first phase a robust and energy aware selection of aggregation nodes using reverse game theory is achieved. The second 


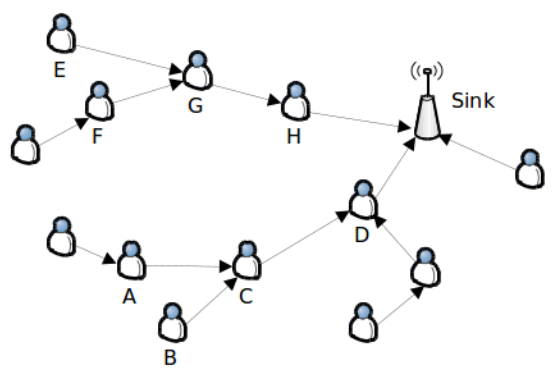

Figure 7: Node $\mathrm{G}$ aggregates correlated data between $\mathrm{E}, \mathrm{F}_{1955}$ and sends it to $\mathrm{H}$ which will deliver it to the sink

phase is associated to an optimized data dissemination and power efficient routing scheme using Ant-colony Optimization. Simulation results indicate remarkable power optimization and enhanced QoS in comparison to $\mathrm{LEACH}$ protocol.

\subsubsection{Discussion and Future Directions}

This domain is strongly related to the routing domain that justifies why game theory has been proposed in only two proposals. In the first one, CAR [119], the cost function takes into account the energy, interference and correlated data. However, the proposal does not pay attention to the end-to-end delay. The othergro [125] divides the routing process into two phases, and uses game theory only in the first phase for a robust and energy aware selection of aggregation nodes.

Many security challenges arises from data aggregation. This is related to the fact that identification infor +975 mation of data is lost once it is aggregated, making the detection of malicious nodes more difficult [126].

Future work should use game theory to address these security challenges. It should focus on the trade-off between energy balancing and delay in both structure ${ }^{+980}$ based and structure-free data aggregation schemes. Besides, comparisons with heuristic based data aggregation proposals [127, 128] are still not covered. Heterogeneous WSNs are suitable scenarios for data aggregation because involving various duties (i.e., relay ${ }^{+985}$ ing, sensing and aggregation) at the same time on a node with limited resources, might quickly drain its battery.

\subsection{Security}

Wireless links in WSNs are susceptible to eavesdropping, impersonating, message modification, Denial of Service $(\overline{D o S})$, among others. Due to the limited capabilities of nodes, researchers had to thinki995 about efficient approaches to solve this problem. Various security challenges and types of attacks in WSNs are analyzed. The key issues that need to be resolved for achieving adequate security are summarized and surveyed in [129, 130].
Many secure routing protocols such as SEAD [131], Ariadne [132], SRP [133], and SAODV [134] are designed for protecting routing information. A misbehaving node could behave well during the route discovery phase, but drop data packets later. Moreover, 550 if misbehaving nodes drop packets, all these solutions can not detect and prevent such attacks, as they focus only on the detection of modification of routing control traffic or fabricating false routing information. With WSNs, security not only has to worry about malicious nodes but also about "selfish nodes". A selfish node is a node that misbehaves, not necessarily because it is a malicious node, but because either it prefers to save its own limited resources or it may belong to a different authority. A big percentage of selfish nodes in a network can rapidly decrease network performance and even create big "blind spots".

Game theory models have been widely used in this domain. A survey of security approaches based on game theory in WSNs is presented in [135]. The recent proposals for achieving energy efficient security algorithms in WSNs based on game theory are discussed below and summarized in Table 8

\subsubsection{Non-cooperative Games}

A proactive defense scheme that uses an evolutionary game theory model is presented in [136]. The concept of evolutionary game theory proposes the player with bounded rationality and dynamics of the game process (i.e., partial knowledge of the state of the game) [137]. The scheme allows nodes to have the ability to learn the evolution of rationality by dynamically adjusting their defense strategies according to the attackers' strategies. The nodes try to find the strategy that best balances their own rewards (i.e., successfully forwarding of data packets) and their costs resulted from deploying the security measures (i.e., energy consumption). The proposed game satisfies the characteristics of the nodes of WSNs. Because the nodes have to consume large amounts of energy if they want to obtain and keep updating the information about the state of the entire network, particularly if the topology changes continuously.

An energy aware Trust Derivation Dilemma Game (TDDG) for WSN-based Internet of Things (IoT) networks is presented in [138]. The work aims to minimize the energy consumption, while maintaining an adequate security level for the WSNs. First, a risk strategy model is presented to stimulate nodes' cooperation. Then, TDDG is introduced into the trust derivation process. Based on the mixed strategy NE, the optimal ratio between the gain and the cost and the probability of the selected strategy are discussed. Simulation results show that the proposed scheme achieves the desirable security and reduces the energy consumption of the network compared with traditional flooding trust derivation mechanisms. 
Coordinator selection is a technique that can considerably defend against attacks and reduce the data delivery delay. In [139], an adaptive coordinator se $\mathrm{se}_{240}$ lection algorithm using game and fuzzy logic is proreliable coordinator to minimize the expected network energy loss. The proposed game model consists of two interrelated formulations: a stochastic game for 045 dynamic defense and a best response policy using evotion. The amount of remaining battery of the selected one is taken into account. The global NE point exists. Then, a mixed-strategy solution for the defender and ${ }_{050}$ the attacker is designed. It combines both evolutionary game NE strategies and stochastic game NE strategies in order to achieve the maximum payoffs for both players.

\subsubsection{Cooperation Enforcement Games}

Trustworthy Energy Efficient Routing (TEER) [140] is an algorithm that aims to distribute energy consumption across sensors evenly, as well as to increase path security in a hierarchical-cluster sensor network. The ${ }^{060}$ cluster heads election process is modeled using game theory. The NE of the game corresponds to the healthier CHs having higher energy and trust level. Firstly, each node establishes a possible head set $\mathcal{P}$ which is empty at the initialization phase. Secondly, each node ${ }^{065}$ will broadcast its own payoff (i.e., $\pi$ value) to all neighbors. After that, each node will compare each neighbor's $\pi$ value with its own. Then it adds the nodes whose $\pi$ value is larger than its own to its possible head set $\mathcal{P}$. If a node's possible head set $\mathcal{P}$ is still empty,o70 this node will declare itself as a $\mathrm{CH}$. The payoff value is calculated using the following formula:

$$
\pi_{i}=\alpha E_{i} / E_{\text {init }}+\beta R_{i}-\gamma \sum P_{\text {pathloss }} /\left(n_{i} P_{\max }\right)
$$

where $\alpha, \beta$ and $\gamma$ denote weight parameters of the node's $i$ residual energy level, trust level, and average path loss within neighbors, correspondingly. $E_{\text {init }}$ denotes node's initial energy level, $E_{i}$ denotes node's current residual energy level, $R_{i}$ denotes node's trus\$oso level, and $\sum P_{\text {pathloss }} /\left(n_{i} P_{\max }\right)$ denotes node's average path loss to its neighbors which can provide cluster head's appropriate position within cluster. Each node tends to elect neighbor node with maximum $\pi$ value as $\mathrm{CH}$ to maximize its payoff. Following this strategy, the 085 energy consumption is distributed and path security is increased. Results indicate that this proposal produces a longer network lifetime and a safer network in comparison to LEACH.

The impact of applying game theory on the networkogo throughput, battery consumption, and accuracy of selfish node detection in WSNs is investigated in [141]. A protocol that is based on game theory is presented. It allows sensors to decide whether or not to forward packets by i) defining a suitable cost and profit for routing and forwarding incoming packets, and ii) keeping a history of experiences with non-cooperating nodes in order to drive selfish nodes out of the WSN. The incentive for each node is to have a better reputation. A node that acts selfishly is the one that randomly drops packets to conserve energy or to corrupt the network intentionally. Over time, nodes with low reputation can be isolated and labeled as "selfish nodes". At each node, there is a trade-off between saving energy resources and maintaining their reputation. The proposal has two main weak points. Firstly, selfish nodes detection is done by the sink which means that the method is totally centralized. Secondly, it is difficult to detect selfish nodes if there is a medium or large number of nodes which have low reputations.

Game-Fuzzy Q-Learning (G-FQL) [142] combines both game theory and fuzzy Q-learning to detect Distributed Denial-of-Service (DDoS) in a cluster-based WSN. DDoS is characterized by the presence of an attacker who sends flooding messages that exhaust nodes' energy in reception and processing. Besides, these messages prevent nodes from entering 'sleep mode'. G-FQL is a triple-player game, in which a cluster head (detector) and the sink (defender) cooperate to provide defense against an attacker. The game has two phases. In the first phase, a $\mathrm{CH}$ (player-1) identifies the level of the attack, that depends on the disruption done by the attacker (player-2), using a fuzzy Q-Learning algorithm. For attacks detection, player-1 adopts three strategies, namely: catch, missed, and low catch. If the level of the attack is above the default value threshold, player-1 $(\mathrm{CH})$ transmits an alarm event containing information about the malicious node to the sink (player3). That information is preprocessed by the sink to travel from phase 1 to 2 . In the second phase, the sink prepares a countermeasure strategy by employing the fuzzy Q-learning algorithm to confirm the malicious node's behavior (i.e., past attacks). The detection player $(\mathrm{CH})$ and defense player (sink) coordinate their defense with each other. Incentive mechanism for cooperation enforcement has been applied. Two constant reward values are defined. $R_{1}$ is the gain of the IDS1 when the $\mathrm{CH}$ detects an attack, and $R_{2}$ is the gain of the IDS 2 when the sink protects the WSN. If the sink does not identify the malicious node during the attack, the reward of the IDS 1 would be $-R_{1}$ (a negative reward). Likewise, if the base station fails to defend the WSN during an attack, the payoff of the IDS2 would be $-R_{2}$. It has been determined that repeated interaction sustains cooperation, builds confidence and enhances reputation. The game has the following utility function:

$$
U=\rho \mathrm{SP}-\beta \mathrm{FN}-\theta \mathrm{FP}
$$

where $\rho$ is the weight of effective prediction, SP is the true confidence rate of attack patterns, $\beta$ is the weight 
of failed estimates (attacks but no defense), $\mathrm{FN}$ is the ${ }_{150}$ false negative of attack patterns (attacks but no defense), $\theta$ denotes the weight of failed predictions (defense but no attack), and FP represents false positive of attack patterns (defense but no attack).

G-FQL algorithm with was compared with existing 155 soft computing methods like Fuzzy Logic Controller (FLC), Q-learning, and Fuzzy Q-learning (FQL), in terms of total energy consumed by sensor nodes and number of alive nodes during the simulation. The results show that the number of alive nodes in G-FQL is s $_{160}$ greater than the other methods. However, a clear conclusion about the performance of G-FQL in term of energy efficiency is missing. It is also important to note that G-FQL is not fully distributed. Only the detection of an attacker is done by clusters in a distributed man $_{2165}$ ner. However, the algorithm is centralized as all the defense actions are done mainly by the sink.

\subsubsection{Discussion and Future Directions}

Due to the nature of the problems in this domain, it is unlikely to see proposals that use cooperative games $_{\dot{2} 170}$ erate we would not have most of the security problems. However, nodes could cooperate in defending against attackers. Nevertheless, we believe that cooperation enforcement mechanisms are preferable in this domain, in the sense that it guarantees cooperation and ${ }_{175}$ makes the defending strategy more robust, as in [142]. Evolutionary game theory [137] is also used in defense models [136, 139]. It does not require a global knowledge of the game state, though it allows the nodes to dynamically adjust their defense strategies taking enz180 ergy consumption costs into account.

Different proposals for security in WSNs that focus on energy efficiency could be found in the literature. eHIP [143] tries to solve the intrusion detection and intrusion prohibition. SEEM [144] uses multi-path com 2185 munications to avoid draining the energy of the nodes of a single path, and avoids that malicious nodes get the traffic routed through themselves. However, these proposals do nothing against selfish nodes. We believe lems of similar nature can not benefit much from the application of game theory.

The main security issue where game theory can really shine is by helping to address problems derived from nodes that misbehave. Specifically in the case 195 where they do it for selfish reasons -as opposed to malicious reasons. We foresee that some of the best future applications of game theory in this domain for energy efficiency and other issues- will use games

2145 that implement reputation schemes, trust models, or at 2200 tack detection and protection mechanisms - just [140], [139, 138, 141] and [142, 136] do, respectively.

An innovative design method of combining game theory and computational intelligence for attack detec- tion and protection has been proposed in [142]. However, the solutions proposed in [140], [141] and [142] are centralized. It would be interesting to research on energy efficient proposals in which the trust model or the protection mechanism are distributed despite the expected challenges that can arise in networks with a large number of nodes.

A recent trend in WSNs is to use data from other WSNs to optimize the operation of a target WSN [145]. For instance, a WSN measuring pollution can use the instantaneous information provided by a WSN measuring the number of cars in a road to adapt its sensing rate to the traffic conditions, thus saving energy when the road is empty. In case the WSNs that exchange data do not belong to the same administrative domain, trustworthiness becomes a fundamental requirement. In this scenario, game theory can help to decide if a WSN may benefit from sharing its data, as well as to guarantee the trustworthiness of the received data.

\subsection{Task allocation}

According to [146], task allocation in WSNs is defined as: (1) the assignment of tasks into sensor nodes, (2) the assignment of communication activities on channels, or (3) the scheduling of computation and communication activities. Recently, task-based systems are needed to provide services to entities outside the network. Allocation of tasks on different wireless nodes must take into account energy constraints, as well as the compatibility of tasks to a given node and/or topology.

Non-game theoretic approaches have been applied in this domain like EcoMapS (Energy-constrained Task Mapping and Scheduling) [147]. EcoMapS is application-independent. It consists of a scheduling system that aims to map and schedule tasks of an application with minimum schedule length subject to consumption constraints in cluster-based WSNs. A different scheduling problem is how to schedule a given set of tasks on a single node, taking into account energy efficiency, as proposed in [148]. In this proposal, the tasks specify an attribute called "importance", also denoted as a power index. It shows the relative importance of a task in relation to the other tasks under low-power conditions. An Integer Linear Programming (ILP) formulation and a polynomial time 3-phase heuristic are proposed in [146] in order to formulate the energy-balanced task allocation onto a cluster-based WSNs. The goal is to find an allocation that maximizes the lifetime of the cluster. Topologyaware energy efficient task assignment for multi-hop WSNs has been addressed in [149], in which an antbased meta-heuristic algorithm was developed to optimize the task assignment. In [150], simulated annealing [151] method was applied to search an optimal task 
assignment, aiming to minimize the total energy consumption and latency. The work described in [152] focuses on a scheduling algorithm for the sub-tasks of an application in WSNs. The goal of the task scheduler there is to maximize the network lifetime. The task scheduling problem is reduced into a min $k$-cut problem (i.e. a well-studied graph problem), which is a polynomial algorithm.

In the following subsections we present and discuss the latest contributions that focus on energy efficiency using game theory in this domain. The proposals are summarized in Table 8

\subsubsection{Cooperation Enforcement Games}

Sensing tasks should be allocated among sensors fairly and in a minimum time. Besides, completing the sensing task faster will also results in energy savings. However, sensors may refuse to execute a task due to their limited energy capabilities. To solve that sensors act selfishly, a Non-cooperative Game Task Scheduling Algorithm (NGTSA) is proposed in [153]. The goal is to find an optimal strategy for splitting main tasks received by the sink into a number of sub-tasks, as well as distributing these subtasks to clusters in the right order. A utility function related to the total task completion time and tasks allocating scheme is designed. ${ }^{226}$ NE is proved. Simulation results illustrate that selfish nodes can be forced to report their true processing capability and endeavor to participate in the measurement. Thereby, the total time for accomplishing the task is minimized and the energy consumption of ${ }^{270}$ nodes is balanced.

In [154], the distributed task allocation problem for multiple concurrent applications in shared WSNs is modeled using a reverse combinatorial auction. In this proposal nodes are models as bidders. Each node bids275 the cost value in terms of available resources (e.g., energy and CPU) for accomplishing the subset of the applications' tasks. Each application may consist of several tasks. The architecture of this scheme is illustrated in Fig. 8. The main objective is to maximize the net 2280 work lifetime by sharing tasks and network resources among applications, while improving the overall QoS (e.g., deadlines) of each application. Since combinatorial reverse auction problem is a NP-complete problem, a heuristic two-phase Winner Determination Proz28s tocol (WDP)is proposed to solve it. In the first phase, a local decision maker is developed to eliminate the subsets of bidders with lower probability of winning. This results in a significantly low overhead for combinatorial auction message exchange. In the second 290 phase, the suboptimal subsets are selected by an ordering heuristic. Simulation experiments are done to evaluate the system efficiency and scalability when the number of concurrent applications and network size increases. The results show a significant difference inz295 terms of energy consumption when the tasks are shared

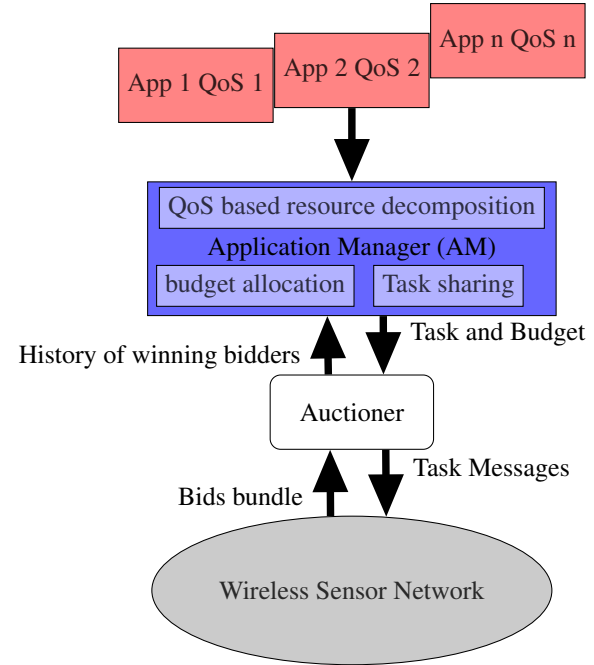

Figure 8: Market based architecture for multiple task allocation

compared to the non-sharing case. Besides, the proposed task allocation scheme outperforms the static energy balanced scheme, Energy Balanced Critical Node Path Three (EB-CNPT) [155], in balancing the energy in the network since the energy level of each sensor is considered in each epoch of the proposed task allocation scheme.

In [156], the work presented in [154] is extended. It claims that given a distributed pool of bids from bidders (i.e., sensor nodes), a centralized Winner Determination Protocol (WDP) would require costly message exchanges with high energy consumption and overhead. Hence, [156] proposes the Energy and Delay Efficient Distributed Winner Determination Protocol (ED-WDP). Simulation results show a fairer energy balance achieved through this bid formulation in comparison to other well-known static schemes. Moreover, in ED-WDP, the message exchange overhead, energy consumption, and delay for winner determination are significantly reduced compared to a centralized WDP.

Few of the previous works notice the constraints on sensors caused by a limited buffer size. Thus, a scheduling policy is proposed in [157]. The scheduler serves first nodes with low remaining energy, as well as those with the least free buffer storage. This solution extends the WSN's lifetime and provides a real-time service quality. It also considers the presence of selfish nodes. It shows their negative impact on the system performance in terms of packet loses, the network lifetime, and spectrum utilization efficiency. Based on a mixed strategy game model, the non-cooperative game converges to an inefficient $\mathrm{NE}$, at which the bandwidth resource is wasted. In order to eliminate user's selfish behavior and enforce cooperation, an incentive mechanism represented by a punishment scheme via a repeated game is added. 


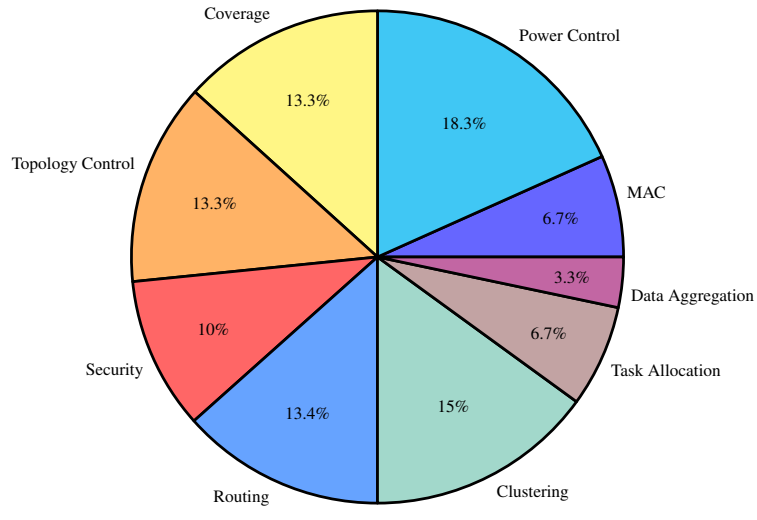

Figure 9: The percentage of latest proposals in each domain of WSNs

\subsubsection{Discussion and Future Directions}

The research in this area is still in its early stages, but it looks very promising. All the proposals in this field apply cooperation enforcement games. [153] aims to distribute sensing tasks between nodes fairly. It deals with nodes' selfish behavior by forcing them to report ${ }^{234}$ their true processing capability. [154, 156] use reverse combinatorial auctions, in which nodes bid the cost value in terms of available resources for accomplishing the subset of the applications' tasks. Since the combinatorial reverse auction problem is a NP-complete problem, a heuristic two-phase winner determination protocol (WDP in [154], ED-WDP in [156]) is proposed to solve it. The difference between WDP and ED-WDP is that the first one is centralized while the other is distributed.

In [157], the selfishness behavior is considered while serving first nodes with the least remaining enand buffer free storage. This proposal also imposes a punishment scheme and to enforce cooperation. It considers the network lifetime maximization ${ }^{235}$ and performance at the same time. Cooperation enforcement models are the best choice for this problem when tasks are independent and can be performed and reported to the server without the cooperation of the other nodes.

In the future work, scenarios that employ nodes of different capabilities should be considered. For example, we can have nodes with multiple sensors that can support several tasks concurrently, or nodes with higher computational capacity that can help others t ${ }^{365}$ execute their tasks.

\subsection{Energy Harvesting WSNs}

Finally, we overview the work done in game the 2370 ory related to Energy Harvesting Wireless Sensor Networks EH-WSNs. Since the number of papers in this area is reduced, we simply describe the papers

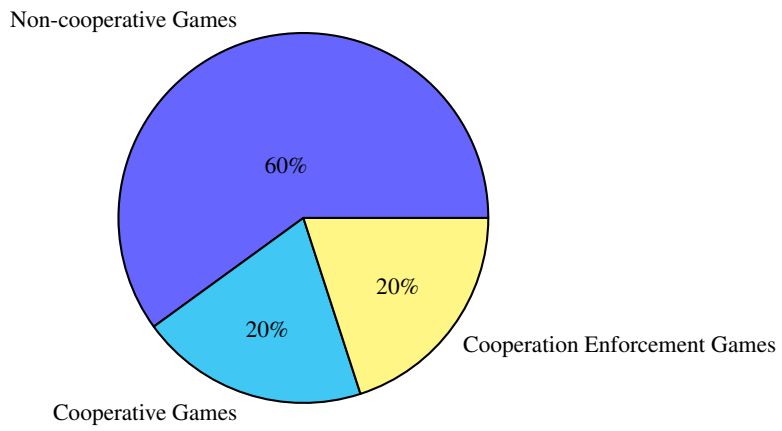

Figure 11: The distribution of the reviewed game theory proposals in the three main classes

in this subsection, including also our thoughts about future research directions in this area.

Energy harvesting technologies comprises a promising solution for WSNs where the battery capacity of sensor nodes is limited and recharging (or replacing) the battery is infeasible. In EH-WSNs, an energy harvesting device (e.g., a solar cell) converts different forms of environmental energy into electricity to be supplied to a sensor node. In this manner, the nodes could prolong lifetime without a need for battery recharge or replacement. However, since it can produce energy only at a limited rate, energy harvesting introduces fundamental issues in the different domains of WSNs. An overview of the various EH research issues, the energy savings mechanisms, and the $\mathrm{EH}$ technologies for WSNs is presented in [51].

Game theory offers tools for solving the various issues of EH-WSNs. In general, the energy level of an energy-harvesting sensor varies dramatically. Hence, distributed estimation of the energy level in EH-WSNs is required. In [158], the unpredictable energy harvested, the battery level, and energy consumption are modeled together in a unified way using game theory. The formulated game has complete and perfect information. The sub-game perfect NE is derived by backward induction. Simulation results show that the proposed model improves the use of the harvested energy and enhances the estimation of the energy level of the nodes.

Another crucial problem in this area that suitable to be addressed using game theory is optimizing the remaining energy of an energy-harvesting sensor. The goal is to satisfy the required QoS at a regular basis under varying amounts of power caused by the ambient or climatic changes (e.g., cloudy or stormy weather).

Finally, the different domains of WSNs (i.e., power control mechanisms, MAC, and routing protocols, among others) should be extended and adapted to cope with the properties and challenges imposed by EH sensors. 


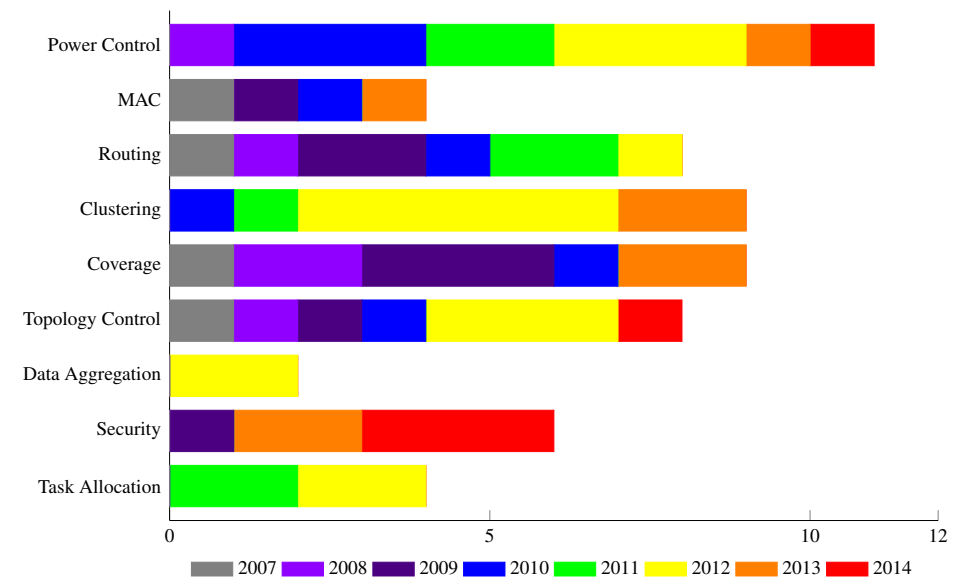

Figure 10: An accumulative chart illustrates the distribution of WSN problems over years

\section{Conclusion}

2375

In this survey we present and discuss the state-of ${ }_{2415}$ the-art of game theory approaches for addressing energy efficiency and lifetime maximization problems in different domains of WSNs like power control, MAC, and routing, among others. We classify the space of games into three main classes: i) non-cooperative, ii $)_{420}$ cooperative, and iii) cooperation enforcement games. We also describe how recent proposals in different WSNs domains employ different classes of games, and how each proposal use the various game theory concepts. Then, the method used by each proposal for achieving energy efficiency and/or lifetime maximization are explained.

Each domain starts with an introduction which presents and discusses the recent work done for addressing the energy efficiency problem in that domain using non-game theory approaches. Then, we present and evaluate the different game theory proposals. At the end of each domain we specify a separate sub 2430 section (called Discussion and Future Directions). It places special emphasis on i) lessons learned in each proposal, and in the domain in general, ii) what is the most appropriate game class for that domain or for specific problems, iii) strength and pitfalls of proposals, and iv) a guide for researchers about gaps that needs to be addressed in the future work.

In addition, comparative tables and statistical charts (see Fig. 9, 10 and 11) are presented to overview how this research area has evolved in the last few years. Fig. 9 and 10 are specially interesting, since they $\mathrm{il}_{2440}$ lustrate the amount of work in each domain over the years. We can notice that the area that attracts most researchers is routing and clustering, followed by power control. This is because of the diversity and importance of the issues that need to be solved in these areas. In contrast, according to the same figures, little amount $\mathrm{n}^{245}$ of work has been done in data aggregation and security. Finally, it is noticeable that the game theory mod- els used for addressing energy efficiency vary from one domain to another, as they depend on the specific problem to address. Thus, it is still hard to categorize the proposals from an energy efficiency point of view.

To conclude, this survey fills a need in the current literature since it covers the latest work in this research area. It is very extensive and thorough, and it will be of invaluable help for any researcher that wants to start contributing to this area.

\section{Acronyms}

WSNs Wireless Sensor Networks

IoT Internet of Things

WLANs Wireless Local Area Networks

MAC Medium Access Control

TDMA Time Division Multiple Access

CDMA Code Division Multiple Access

CWmin minimum Contention Window

FSR Frame Access Rate

SINR Signal Interference plus Noise Ratio

QoS Quality of Service

CSMA Carrier Sense Multiple Access

MER Minimum Energy Routing

IDS Intrusion Detection System

TC Topology Control

LP Linear Programming

NE Nash equilibrium

BNE Bayesian Nash equilibrium

EPG Exact Potential Game

OPG Ordinal Potential Game

NBS Nash Bargaining Solution

DAP Deferred Acceptance Procedure

DoS Denial of Service

WSLS Win-Stay, Lose-Shift

ILP Integer Linear Programming

EH-WSNs Energy Harvesting Wireless Sensor Networks 


\section{References}

[1] J. Yick, B. Mukherjee, D. Ghosal, Wireless sensor network survey, Computer networks 52 (12) (2008) 2292-2330.

[2] T. Rault, A. Bouabdallah, Y. Challal, Energy-efficiency in wireless sensor networks: A top-down review approach, Computer Networks.

[3] N. Nisan, Algorithmic game theory, Cambridge University Press, 2007.

[4] M. Felegyhazi, J.-P. Hubaux, Game theory in wireless networks: A tutorial, Tech. rep., Technical Report LCAREPORT-2006-002, EPFL (2006).

[5] A. B. MacKenzie, L. A. DaSilva, Game theory for wireless engineers, Synthesis Lectures on Communications 1 (1) (2006) 1-86.

[6] R. Machado, S. Tekinay, A survey of game-theoretic approaches in wireless sensor networks, Computer Networks ${ }_{535}$ 52 (16) (2008) 3047-3061.

[7] H.-Y. Shi, W.-L. Wang, N.-M. Kwok, S.-Y. Chen, Game theory for wireless sensor networks: A survey, Sensors 12 (7) (2012) 9055-9097.

[8] V. Srivastava, J. Neel, A. MacKenzie, R. Menon, L. Dasilva 2540 J. Hicks, J. Reed, R. Gilles, Using game theory to analyze wireless ad hoc networks, Communications Surveys Tutorials, IEEE 7 (4) (2005) 46-56. doi : 10.1109/COMST . 2005. 1593279

[9] I. F. Akyildiz, W. Su, Y. Sankarasubramaniam, E. Cayirci ${ }_{2545}$ Wireless sensor networks: A survey, Computer networks 38 (4) (2002) 393-422.

[10] H. Karl, A. Willig, Protocols and architectures for wireless sensor networks, Wiley. com, 2007.

[11] D. E. Charilas, A. D. Panagopoulos, A survey on game the $\overline{\overline{2}}_{550}$ ory applications in wireless networks, Computer Networks 54 (18) (2010) 3421-3430.

[12] J. Nash, Non-cooperative games, Annals of mathematics (1951) 286-295.

[13] D. Monderer, L. S. Shapley, Potential games, Games and $\operatorname{eco}_{\overline{2} 555}$ nomic behavior 14 (1) (1996) 124-143.

[14] L. S. Shapley, A value for n-person games, Tech. rep., DTIC Document (1952)

[15] J. F. Nash Jr, The bargaining problem, Econometrica: Journal of the Econometric Society (1950) 155-162.

[16] R. J. Aumann, Correlated equilibrium as an expression of bayesian rationality, Econometrica: Journal of the Econometric Society (1987) 1-18.

[17] N. Pantazis, D. Vergados, A survey on power control issues in wireless sensor networks, Communications Surveys $\mathrm{Tu}_{\overline{2} 565}$ torials, IEEE 9 (4) (2007) 86-107. doi:10.1109/COMST . 2007.4444752

[18] M. Kubisch, H. Karl, A. Wolisz, L. C. Zhong, J. Rabaey, Distributed algorithms for transmission power control in wireless sensor networks, in: Wireless Communications and Network $\overline{\overline{2}}_{570}$ ing, 2003. WCNC 2003. 2003 IEEE, Vol. 1, IEEE, 2003, pp. 558-563.

[19] M. Chiang, C. W. Tan, D. P. Palomar, D. O’Neill, D. Julian, Power control by geometric programming, Wireless Communications, IEEE Transactions on 6 (7) (2007) 2640-2651. 257

[20] S. Lin, J. Zhang, G. Zhou, L. Gu, J. A. Stankovic, T. He, Atpc: Adaptive Transmission Power Control for wireless sensor networks, in: Proceedings of the 4th international conference on Embedded networked sensor systems, ACM, 2006, pp. 223-236.

[21] S. Sengupta, M. Chatterjee, K. A. Kwiat, A game theoretic framework for power control in wireless sensor networks, Computers, IEEE Transactions on 59 (2) (2010) 231-242.

[22] G. Zheng, Study on the power control of wireless sensor networks based on game theory, Journal of Information and $\mathrm{z}_{585}$ Computational Science 7 (2010) 957-964.

[23] F.-Y. Tsuo, H.-P. Tan, Y. H. Chew, H.-Y. Wei, Energy-aware transmission control for wireless sensor networks powered by ambient energy harvesting: A game-theoretic approach, in:
Communications (ICC), 2011 IEEE International Conference on, IEEE, 2011, pp. 1-5.

[24] W. Tushar, D. Smith, T. A. Lamahewa, J. Zhang, Noncooperative power control game in a multi-source wireless sensor network, in: Communications Theory Workshop (AusCTW), 2012 Australian, IEEE, 2012, pp. 43-48.

[25] J. Luo, C. Pan, R. Li, F. Ge, Power control in distributed wireless sensor networks based on noncooperative game theory, International Journal of Distributed Sensor Networks (2012) 10 .

[26] B. Chai, R. Deng, P. Cheng, J. Chen, Energy-efficient power allocation in cognitive sensor networks: A game theoretic approach, in: Global Communications Conference (GLOBECOM), 2012 IEEE, 2012, pp. 416-421.

[27] J. Jia, Q. Zhang, A non-cooperative power control game for secondary spectrum sharing, in: Communications, 2007. ICC'07. IEEE International Conference on, IEEE, 2007, pp. 5933-5938.

[28] X.-C. Hao, Q.-Q. Gong, S. Hou, B. Liu, Joint channel allocation and power control optimal algorithm based on noncooperative game in wireless sensor networks, Wireless Personal Communications (2014) 1-15.

[29] S.-S. Byun, I. Balasingham, Power control for mission critical wireless sensor networks using repeated coalitional games, in: Military Communications Conference, 2008. MILCOM 2008. IEEE, IEEE, 2008, pp. 1-7.

[30] F. Meshkati, H. V. Poor, S. C. Schwartz, Energy-efficient resource allocation in wireless networks, Signal Processing Magazine, IEEE 24 (3) (2007) 58-68.

[31] S. K. Jayaweera, K. Hakim, A cooperative game theoretic solution for lifetime maximization of WSNs in sequential estimation, in: Information and Automation for Sustainability (ICIAFs), 2010 5th International Conference on, IEEE, 2010, pp. 233-238.

[32] F. Kazemeyni, E. B. Johnsen, O. Owe, I. Balasingham, Group selection by nodes in wireless sensor networks using coalitional game theory, in: Engineering of Complex Computer Systems (ICECCS), 2011 16th IEEE International Conference on, IEEE, 2011, pp. 253-262.

[33] M. Clavel, F. Durán, S. Eker, P. Lincoln, N. Martı-Oliet, J. Meseguer, J. F. Quesada, Maude: Specification and programming in rewriting logic, Theoretical Computer Science 285 (2) (2002) 187-243.

[34] X.-N. Miao, G. Xu, Cooperative differential game model based on trade-off between energy and delay for wireless sensor networks, Annals of Operations Research (2013) 1-14.

[35] C. Cano, B. Bellalta, A. Sfairopoulou, M. Oliver, Low energy operation in wsns: A survey of preamble sampling MAC protocols, Computer Networks 55 (15) (2011) 3351-3363.

[36] M. Buettner, G. V. Yee, E. Anderson, R. Han, X-MAC: a short preamble MAC protocol for duty-cycled wireless sensor networks, in: Proceedings of the 4th international conference on Embedded networked sensor systems, ACM, 2006, pp. 307320.

[37] J. Polastre, J. Hill, D. Culler, Versatile low power media access for wireless sensor networks, in: Proceedings of the 2nd international conference on Embedded networked sensor systems, ACM, 2004, pp. 95-107.

[38] T. Van Dam, K. Langendoen, An adaptive energy-efficient MAC protocol for wireless sensor networks, in: Proceedings of the 1st international conference on Embedded networked sensor systems, ACM, 2003, pp. 171-180.

[39] W. Ye, J. Heidemann, D. Estrin, Medium access control with coordinated adaptive sleeping for wireless sensor networks, Networking, IEEE/ACM Transactions on 12 (3) (2004) 493506.

[40] L. F. van Hoesel, P. J. Havinga, A lightweight medium access protocol (LMAC) for wireless sensor networks: Reducing preamble transmissions and transceiver state switches.

[41] I. Rhee, A. Warrier, M. Aia, J. Min, M. L. Sichitiu, Z-MAC: a hybrid MAC for wireless sensor networks, IEEE/ACM Trans- 
actions on Networking (TON) 16 (3) (2008) 511-524. 2660

[42] K. Langendoen, A. Meier, Analyzing MAC protocols for low data-rate applications, ACM Transactions on Sensor Networks (TOSN) 7 (2) (2010) 19.

[43] C. Cano, B. Bellalta, A. Sfairopoulou, J. Barceló, A low power listening MAC with scheduled wake up after trans2665 missions for WSNs, Communications Letters, IEEE 13 (4) (2009) 221-223.

[44] M. Felegyhazi, J.-P. Hubaux, Game theory in wireless networks: A tutorial, Tech. rep., Technical Report LCAREPORT-2006-002, EPFL (2006).

[45] J. Barcelo, H. Inaltekin, B. Bellalta, Obey or play: asymptotic equivalence of slotted aloha with a game theoretic contention model, Communications Letters, IEEE 15 (6) (2011) 623625.

[46] J. Konorski, A game-theoretic study of CSMA/CA under675 a backoff attack, IEEE/ACM Transactions on Networking (TON) 14 (6) (2006) 1167-1178.

[47] J. Konorski, A station strategy to deter backoff attacks in IEEE 802.11 LANs, Journal of discrete Algorithms 5 (3) (2007) 436-454.

[48] L. Zhao, L. Guo, L. Cong, H. Zhang, An energy-efficient MAC protocol for WSNs: Game-theoretic constraint optimization with multiple objectives, Wireless Sensor Networks 1 (2009) 358-364

[49] J. K. Park, J. Ha, H. Seo, J. Kim, C. W. Choi, Stability o $6_{685}$ game-theoretic energy-aware MAC scheme for wireless sensor networks, in: Sensor Networks, Ubiquitous, and Trustworthy Computing (SUTC), 2010 IEEE International Conference on, IEEE, 2010, pp. 384-389.

[50] A. Abrardo, L. Balucanti, A. Mecocci, A game theory diss690 tributed approach for energy optimization in wsns, ACM Transactions on Sensor Networks (TOSN) 9 (4) (2013) 44.

[51] D. Niyato, E. Hossain, M. M. Rashid, V. K. Bhargava, Wireless sensor networks with energy harvesting technologies: a game-theoretic approach to optimal energy management;695 Wireless Communications, IEEE 14 (4) (2007) 90-96.

[52] W. Guo, W. Zhang, A survey on intelligent routing protocols in wireless sensor networks, Journal of Network and Computer Applications 38 (2014) 185-201.

[53] R. V. Kulkarni, A. Forster, G. K. Venayagamoorthy, Compuz700 tational intelligence in wireless sensor networks: A survey, Communications Surveys \& Tutorials, IEEE 13 (1) (2011) 68-96.

[54] B. Arisian, K. Eshghi, A game theory approach for optimal routing: In wireless sensor networks, in: Wireless Communiz705 cations Networking and Mobile Computing (WiCOM), 2010 6th International Conference on, IEEE, 2010, pp. 1-7.

[55] Z. Jia, M. Chundi, H. Jianbin, Game theoretic energy balance routing in wireless sensor networks, in: Control Conference, 2007. CCC 2007. Chinese, IEEE, 2007, pp. 420-424. 271

[56] K.-H. Han, Y.-B. Ko, J.-H. Kim, A novel gradient approach for efficient data dissemination in wireless sensor networks, in: Vehicular Technology Conference, 2004. VTC2004-Fall. 2004 IEEE 60th, Vol. 4, IEEE, 2004, pp. 2979-2983.

[57] A. Schillings, K. Yang, VGTR: A collaborative, energy and 715 information aware routing algorithm for wireless sensor networks through the use of game theory, in: GeoSensor Networks, Springer, 2009, pp. 51-62.

[58] C. Intanagonwiwat, R. Govindan, D. Estrin, Directed diffusion: a scalable and robust communication paradigm foz720 sensor networks, in: Proceedings of the 6th annual international conference on Mobile computing and networking, ACM, 2000, pp. 56-67.

[59] G. P. Hancke, C. J. Leuschner, et al., SEER: a simple energy efficient routing protocol for wireless sensor networks. 2725

[60] R. C. Shah, J. M. Rabaey, Energy aware routing for low energy ad hoc sensor networks, in: Wireless Communications and Networking Conference, 2002. WCNC2002. 2002 IEEE, Vol. 1, IEEE, 2002, pp. 350-355.

[61] W. R. Heinzelman, A. Chandrakasan, H. Balakrishnanz730
Energy-efficient communication protocol for wireless microsensor networks, in: System Sciences, 2000. Proceedings of the 33rd Annual Hawaii International Conference on, IEEE, 2000, pp. 10-pp.

[62] M. Zheng, Game theory used for reliable routing modeling in wireless sensor networks, in: Parallel and Distributed Computing, Applications and Technologies (PDCAT), 2010 International Conference on, IEEE, 2010, pp. 280-284.

[63] D. B. Johnson, D. A. Maltz, J. Broch, et al., DSR: The dynamic source routing protocol for multi-hop wireless ad hoc networks, Ad hoc networking 5 (2001) 139-172.

[64] A. Behzadan, A. Anpalagan, B. Ma, Prolonging network lifetime via nodal energy balancing in heterogeneous wireless sensor networks, in: Communications (ICC), 2011 IEEE International Conference on, IEEE, 2011, pp. 1-5.

[65] N. Sadagopan, M. Singh, B. Krishnamachari, Decentralized utility-based sensor network design, Mobile Networks and Applications 11 (3) (2006) 341-350.

[66] D. Sun, X. Huang, Y. Liu, H. Zhong, Predictable energy aware routing based on dynamic game theory in wireless sensor networks, Computers \& Electrical Engineering.

[67] T. Wu, K. Yue, W. Liu, J. Xu, An energy-efficient data transfer model of wireless sensor networks based on the coalitional game theory, in: Fuzzy Systems and Knowledge Discovery (FSKD), 2011 Eighth International Conference on, Vol. 3, IEEE, 2011, pp. 1354-1358.

[68] C. Pandana, Z. Han, K. R. Liu, Cooperation enforcement and learning for optimizing packet forwarding in autonomous wireless networks, Wireless Communications, IEEE Transactions on 7 (8) (2008) 3150-3163.

[69] C.-k. Lin, et al., Energy-aware path selection in mobile wireless sensor networks: A dynamic bayesian game approach, in: Personal, Indoor and Mobile Radio Communications, 2009 IEEE 20th International Symposium on, IEEE, 2009, pp. 1198-1203.

[70] C. E. Perkins, E. M. Royer, Ad-hoc on-demand distance vector routing, in: Mobile Computing Systems and Applications, 1999. Proceedings. WMCSA'99. Second IEEE Workshop on, IEEE, 1999, pp. 90-100.

[71] A. Manjeshwar, D. P. Agrawal, TEEN: A routing protocol for enhanced efficiency in wireless sensor networks, in: IPDPS, Vol. 1, 2001, p. 189.

[72] A. Manjeshwar, D. P. Agrawal, APTEEN: A hybrid protocol for efficient routing and comprehensive information retrieval in wireless sensor networks, in: ipdps, Vol. 2, 2002, p. 48.

[73] D. Lee, H. Shin, C. Lee, Game theory-based resource allocation strategy for clustering based wireless sensor network, in: Proceedings of the 6th International Conference on Ubiquitous Information Management and Communication, ACM, 2012, p. 112

[74] X. Wu, G. Chen, S. Das, Avoiding energy holes in wireless sensor networks with nonuniform node distribution, Parallel and Distributed Systems, IEEE Transactions on 19 (5) (2008) 710-720. doi:10.1109/TPDS. 2007.70770

[75] A.-F. Liu, P.-H. Zhang, Z.-G. Chen, Theoretical analysis of the lifetime and energy hole in cluster based wireless sensor networks, Journal of Parallel and Distributed Computing 71 (10) (2011) 1327-1355.

[76] Z. Xu, Y. Yin, J. Wang, J.-U. Kim, A density-based energyefficient game-theoretic routing algorithm for wireless sensor networks.

[77] Y. Yang, C. Lai, L. Wang, X. Wang, An energy-aware clustering algorithm via game theory for wireless sensor networks, in: Control, Automation and Systems (ICCAS), 2012 12th International Conference on, 2012, pp. 261-266.

[78] J. Wu, R. Axelrod, How to cope with noise in the iterated prisoner's dilemma, Journal of Conflict resolution (1995) 183189.

[79] X.-H. Lin, Y.-K. Kwok, H. Wang, N. Xie, A game theoretic approach to balancing energy consumption in heterogeneous wireless sensor networks, Wireless Communications and Mo- 
bile Computing.

[80] H. Jing, H. Aida, Cooperative clustering algorithms for wireless sensor networks, cite from Smart Wireless Sensor Networks InTech (2010) 157-172.

[81] L. Tan, S. Zhang, J. QI, Cooperative cluster head selection based on cost sharing game for energy-efficient wireless sensor networks, Journal of Computational Information Systems 8 (9) (2012) 3623-3633.

[82] A. C. Voulkidis, M. P. Anastasopoulos, P. G. Cottis, Energye810 efficiency in wireless sensor networks: A game-theoretic approach based on coalition formation, ACM Transactions on Sensor Networks (TOSN) 9 (4) (2013) 43.

[83] Y. Zeng, Z. Chen, C. Qiao, L. Xu, A cluster header election scheme based on auction mechanism for intrusion detections15 in MANET, in: Network Computing and Information Security (NCIS), 2011 International Conference on, Vol. 2, IEEE, 2011, pp. 433-437.

[84] J. Chen, H. Huang, R. J. Kauffman, A public procurement combinatorial auction mechanism with quality assignment2,820 Decision Support Systems 51 (3) (2011) 480-492.

[85] J. Zheng, M. Z. A. Bhuiyan, S. Liang, X. Xing, G. Wang, Auction-based adaptive sensor activation algorithm for target tracking in wireless sensor networks, Future Generation Computer Systems.

[86] S. Meguerdichian, F. Koushanfar, M. Potkonjak, M. B. Srivastava, Coverage problems in wireless ad-hoc sensor networks, in: in IEEE INFOCOM, 2001, pp. 1380-1387.

[87] C. T. Vu, Distributed energy-efficient solutions for area coverage problems in wireless sensor networks, Computer Sciencesзo Dissertations (2009) 37

[88] H. Zhang, J. C. Hou, On the upper bound of $\alpha$-lifetime for large sensor networks, ACM Transactions on Sensor Networks (TOSN) 1 (2) (2005) 272-300.

[89] S. Slijepcevic, M. Potkonjak, Power efficient organization o£835 wireless sensor networks, in: Communications, 2001. ICC 2001. IEEE International Conference on, Vol. 2, IEEE, 2001, pp. $472-476$.

[90] Z. Abrams, A. Goel, S. Plotkin, Set k-cover algorithms for energy efficient monitoring in wireless sensor networks, in2840 Proceedings of the 3rd international symposium on Information processing in sensor networks, ACM, 2004, pp. 424-432.

[91] C.-F. Huang, Y.-C. Tseng, The coverage problem in a wireless sensor network, Mobile Networks and Applications 10 (4) (2005) 519-528

92] X.-Y. Li, P.-J. Wan, O. Frieder, Coverage in wireless ad hoc sensor networks, Computers, IEEE Transactions on 52 (6) (2003) 753-763.

[93] X. Ai, V. Srinivasan, C.-K. Tham, DRACo: Distributed, robust an asynchronous coverage in wireless sensor networks 2850 in: Sensor, Mesh and Ad Hoc Communications and Networks, 2007. SECON'07. 4th Annual IEEE Communications Society Conference on, IEEE, 2007, pp. 530-539.

[94] W. Saad, Z. Han, M. Debbah, A. Hjorungnes, T. Basar, Coalitional game theory for communication networks, Signal Proz855 cessing Magazine, IEEE 26 (5) (2009) 77-97.

[95] X. Ai, V. Srinivasan, C.-K. Tham, Optimality and complexity of pure nash equilibria in the coverage game, Selected Areas in Communications, IEEE Journal on 26 (7) (2008) 11701182.

[96] X. He, X. Gui, The localized area coverage algorithm based on game-theory for WSN, Journal of Networks 4 (10) (2009) 1001-1008.

[97] L. Zhang, Y. Lu, L. Chen, D. Dong, Game theoretical algorithm for coverage optimization in wireless sensor netz865 works, in: Proceedings of the World Congress on Engineering, Vol. 1, 2008, pp. 2-4.

[98] L. Zhang, K. Axhausen, D. Ou, Y. Lu, L. Chen, A cognitive approach to link optimization utilized in wireless sensor networks, in: Proceedings of 2009 18th World Imacs870 Congress and Modsim09 International Congress on Modelling and Simulation: Interfacing Modelling and Simulation with Mathematical and Computational Sciences, 2009.

[99] P. Santi, The critical transmitting range for connectivity in mobile ad hoc networks, Mobile Computing, IEEE Transactions on 4 (3) (2005) 310-317.

[100] M. Zhu, S. Martinez, Distributed coverage games for energyaware mobile sensor networks, SIAM Journal on Control and Optimization 51 (1) (2013) 1-27.

[101] D. Monderer, L. S. Shapley, Potential games, Games and economic behavior 14 (1) (1996) 124-143.

[102] M. Zhu, S. Martinez, Distributed coverage games for mobile visual sensors (i): Reaching the set of nash equilibria, in: Decision and Control, 2009 held jointly with the 2009 28th Chinese Control Conference. CDC/CCC 2009. Proceedings of the 48th IEEE Conference on, IEEE, 2009, pp. 169-174.

[103] M. Zhu, S. Martinez, Distributed coverage games for mobile visual sensors (ii): Reaching the set of global optima, in: Decision and Control, 2009 held jointly with the 2009 28th Chinese Control Conference. CDC/CCC 2009. Proceedings of the 48th IEEE Conference on, IEEE, 2009, pp. 175-180.

[104] C. D. Truong, M. A. Khan, F. Sivrikaya, S. Albayrak, Cooperative game theoretic approach to energy-efficient coverage in wireless sensor networks, in: Networked Sensing Systems (INSS), 2010 Seventh International Conference on, IEEE, 2010, pp. 73-76.

[105] I. Ben Abid, N. Boudriga, Game theory for misbehaving detection in wireless sensor networks, in: Information Networking (ICOIN), 2013 International Conference on, IEEE, 2013, pp. 60-65.

[106] W. Xu, W. Trappe, Y. Zhang, Channel surfing: defending wireless sensor networks from interference, in: Proceedings of the 6th international conference on Information processing in sensor networks, ACM, 2007, pp. 499-508.

[107] A. Aziz, Y. Sekercioglu, P. Fitzpatrick, M. Ivanovich, A survey on distributed topology control techniques for extending the lifetime of battery powered wireless sensor networks.

[108] M. Younis, I. F. Senturk, K. Akkaya, S. Lee, F. Senel, Topology management techniques for tolerating node failures in wireless sensor networks: A survey, Computer Networks 58 (2014) 254-283.

[109] R. S. Komali, A. B. MacKenzie, R. P. Gilles, Effect of selfish node behavior on efficient topology design, Mobile Computing, IEEE Transactions on 7 (9) (2008) 1057-1070.

[110] R. Wattenhofer, L. Li, P. Bahl, Y.-M. Wang, Distributed topology control for power efficient operation in multihop wireless ad hoc networks, in: INFOCOM 2001. Twentieth Annual Joint Conference of the IEEE Computer and Communications Societies. Proceedings. IEEE, Vol. 3, IEEE, 2001, pp. 1388-1397.

[111] R. S. Komali, A. B. MacKenzie, Distributed topology control in ad-hoc networks: A game theoretic perspective, in: Proceedings of IEEE CCNC, 2006, pp. 563-568.

[112] P. Closas, J. A. Fernandez-Rubio, et al., A game theoretical algorithm for joint power and topology control in distributed WSN, in: Acoustics, Speech and Signal Processing, 2009. ICASSP 2009. IEEE International Conference on, IEEE, 2009, pp. 2765-2768.

[113] H. Ren, M.-H. Meng, Game-theoretic modeling of joint topology control and power scheduling for wireless heterogeneous sensor networks, Automation Science and Engineering, IEEE Transactions on 6 (4) (2009) 610-625.

[114] X.-C. Hao, Y.-X. Zhang, N. Jia, B. Liu, Virtual game-based energy balanced topology control algorithm for wireless sensor networks, Wireless Personal Communications (2012) 120.

[115] S. Zarifzadeh, N. Yazdani, Neighbor selection game in wireless ad hoc networks, Wireless Personal Communications (2012) 1-24.

[116] X. Chu, H. Sethu, Cooperative topology control with adaptation for improved lifetime in wireless ad hoc networks, in: INFOCOM, 2012 Proceedings IEEE, IEEE, 2012, pp. 262270. 
[117] I. F. Senturk, K. Akkaya, S. Yilmaz, Relay placement for restoring connectivity in partitioned wireless sensor network8945 under limited information, Ad Hoc Networks 13 (2014) 487503.

[118] O. Namvar Gharehshiran, V. Krishnamurthy, On prolonging life-time in wireless sensor networks with application in localization: A coalitional game-theoretic approach, in: Acousz950 tics Speech and Signal Processing (ICASSP), 2010 IEEE International Conference on, IEEE, 2010, pp. 2874-2877.

[119] E. Zeydan, D. Kivanc, C. Comaniciu, U. Tureli, Energyefficient routing for correlated data in wireless sensor networks, Ad Hoc Networks 10 (6) (2012) 962-975.

[120] R. Rajagopalan, P. Varshney, Data-aggregation techniques in sensor networks: A survey, Communications Surveys Tutorials, IEEE 8 (4) (2006) 48-63. doi : 10.1109/COMST . 2006. 283821

[121] C. Schurgers, M. B. Srivastava, Energy efficient routing960 in wireless sensor networks, in: Military Communications Conference, 2001. MILCOM 2001. Communications for Network-Centric Operations: Creating the Information Force. IEEE, Vol. 1, IEEE, 2001, pp. 357-361.

[122] S. Lindsey, C. S. Raghavendra, PEGASIS: Power-efficien\$965 gathering in sensor information systems, in: Aerospace conference proceedings, 2002. IEEE, Vol. 3, IEEE, 2002, pp. 31125.

[123] C.-M. Chao, T.-Y. Hsiao, Design of structure-free and energybalanced data aggregation in wireless sensor networks, Jourz97 nal of Network and Computer Applications 37 (2014) 229239.

[124] P. Von Rickenbach, R. Wattenhofer, Gathering correlated data in sensor networks, in: Proceedings of the 2004 joint workshop on Foundations of mobile computing, ACM, 2004, pp2975 60-66.

[125] M. Bharathi, B. V. Kumar, Reverse game theory approach for aggregator nodes selection with ant colony optimization based routing in wireless sensor network.

[126] M. Guerrero-Zapata, R. Zilan, J. M. Barceló-Ordinas, K. Bizgso cakci, B. Tavli, The future of security in wireless multimedia sensor networks, Telecommunication Systems 45 (1) (2010) 77-91.

[127] W.-H. Liao, Y. Kao, C.-M. Fan, Data aggregation in wireless sensor networks using ant colony algorithm, Journal of Net2985 work and Computer Applications 31 (4) (2008) 387-401.

[128] C. Intanagonwiwat, D. Estrin, R. Govindan, J. Heidemann, Impact of network density on data aggregation in wireless sensor networks, in: Distributed Computing Systems, 2002. Proceedings. 22nd International Conference on, IEEE, 2002,2990 pp. 457-458.

[129] F. Hu, N. K. Sharma, Security considerations in ad hoc sensor networks, Ad Hoc Networks 3 (1) (2005) 69-89.

[130] Y. Zhou, Y. Fang, Y. Zhang, Securing wireless sensor networks: A survey, Communications Surveys Tutorials, IEEE2995 10 (3) (2008) 6-28. doi:10.1109/COMST . 2008.4625802

[131] Y.-C. Hu, D. B. Johnson, A. Perrig, SEAD: Secure efficient distance vector routing for mobile wireless ad hoc networks, Ad Hoc Networks 1 (1) (2003) 175-192.

[132] Y.-C. Hu, A. Perrig, D. B. Johnson, Ariadne: A secure onзо00 demand routing protocol for ad hoc networks, Wireless Networks 11 (1-2) (2005) 21-38.

[133] K. Sanzgiri, B. Dahill, B. N. Levine, C. Shields, E. M Belding-Royer, A secure routing protocol for ad hoc networks, in: Network Protocols, 2002. Proceedings. 10th IEEF3005 International Conference on, IEEE, 2002, pp. 78-87.

[134] M. G. Zapata, Secure ad hoc on-demand distance vector routing, ACM SIGMOBILE Mobile Computing and Communications Review 6 (3) (2002) 106-107.

[135] S. Shen, G. Yue, Q. Cao, F. Yu, A survey of game theory ir3010 wireless sensor networks security, Journal of Networks 6 (3) (2011) 521-532.

[136] Z. Chen, C. Qiao, Y. Qiu, L. Xu, W. Wu, Dynamics stability in wireless sensor networks active defense model, Journal of
Computer and System Sciences 2014

[137] J. Hofbauer, K. Sigmund, Evolutionary game dynamics, Bulletin of the American Mathematical Society 40 (4) (2003) 479-519.

[138] J. Duan, D. Gao, D. Yang, C. H. Foh, H.-H. Chen, An energyaware trust derivation scheme with game theoretic approach in wireless sensor networks for IoT applications 2014

[139] J. Liu, G. Yue, S. Shen, H. Shang, H. Li, A game-theoretic response strategy for coordinator attack in wireless sensor networks, The Scientific World Journal 2014.

[140] J.-t. Wang, Z.-g. Chen, X.-h. Deng, A trustworthy energyefficient routing algorithm based on game-theory for WSN.

[141] M. Asadi, C. Zimmerman, A. Agah, A game-theoretic approach to security and power conservation in wireless sensor networks, International Journal of Network Security 15 (1) (2013) 50-58.

[142] S. Shamshirband, A. Patel, N. B. Anuar, M. L. M. Kiah, A. Abraham, Cooperative game theoretic approach using fuzzy Q-learning for detecting and preventing intrusions in wireless sensor networks, Engineering Applications of Artificial Intelligence.

[143] W.-T. Su, K.-M. Chang, Y.-H. Kuo, eHIP: An energyefficient hybrid intrusion prohibition system for cluster-based wireless sensor networks Computer Networks 51 (4) (2007) 1151 - 1168. doi:http: //dx.doi.org/10.1016/j.comnet.2006.07.008 URL http://www.sciencedirect.com/science/ article/pii/S1389128606002015

[144] N. Nasser, Y. Chen, SEEM: Secure and energy-efficient multipath routing protocol for wireless sensor networks Computer Communications 30 (1112) (2007) 2401 - 2412, ¡ce:title $\_$Special issue on security on wireless ad hoc and sensor networks;/ce:title $i$. doi:http: //dx.doi.org/10.1016/j.comcom.2007.04.014 URL http://www.sciencedirect.com/science/ article/pii/S0140366407001727

[145] S. Pal, S. Oechsner, B. Bellalta, M. Oliver, Performance optimization of multiple interconnected heterogeneous sensor networks via collaborative information sharing, Journal of Ambient Intelligence and Smart Environments 5 (4) (2013) 403-413.

[146] Y. Yu, V. K. Prasanna, Energy-balanced task allocation for collaborative processing in wireless sensor networks, Mobile Networks and Applications 10 (1-2) (2005) 115-131.

[147] Y. Tian, E. Ekici, F. Ozguner, Energy-constrained task mapping and scheduling in wireless sensor networks, in: Mobile Adhoc and Sensor Systems Conference, 2005. IEEE International Conference on, IEEE, 2005, pp. 8-pp.

[148] F. Delicato, F. Protti, J. F. De Rezende, L. Rust, L. Pirmez, Application-driven node management in multihop wireless sensor networks, in: Networking-ICN 2005, Springer, 2005, pp. $569-576$

[149] B. Zhao, M. Wang, Z. Shao, J. Cao, K. C. Chan, J. Su, Topology-aware energy efficient task assignment for collaborative in-network processing in distributed sensor systems, in: Distributed Embedded Systems: Design, Middleware and Resources, Springer, 2008, pp. 201-211.

[150] H. Park, M. B. Srivastava, Energy-efficient task assignment framework for wireless sensor networks.

[151] D. Henderson, S. H. Jacobson, A. W. Johnson, The theory and practice of simulated annealing, in: Handbook of metaheuristics, Springer, 2003, pp. 287-319.

[152] A. Voinescu, D. S. Tudose, N. Tapus, Task scheduling in wireless sensor networks, in: Networking and Services (ICNS), 2010 Sixth International Conference on, IEEE, 2010, pp. 12-17.

[153] L. Dai, Y. Chang, Z. Shen, L. Dai, Y. Chang, Z. Shen, Noncooperative game algorithm for task scheduling in wireless sensor networks, Int. J. Comput. Commun. Contr 6 (2011) 592-602.

[154] N. Edalat, W. Xiao, N. Roy, S. K. Das, M. Motani, Combina- 
torial auction-based task allocation in multi-application wireless sensor networks, in: Embedded and Ubiquitous Computing (EUC), 2011 IFIP 9th International Conference on, IEEE, 2011, pp. 174-181.

[155] Y. Tian, Y. Gu, E. Ekici, F. Ozguner, Dynamic critical-path task mapping and scheduling for collaborative in-network processing in multi-hop wireless sensor networks, in: Parallel Processing Workshops, 2006. ICPP 2006 Workshops. 2006 International Conference on, IEEE, 2006, pp. 8-pp.

[156] N. Edalat, C.-K. Tham, W. Xiao, An auction-based strategy for distributed task allocation in wireless sensor networks Computer Communications 35 (8) (2012) 916 - 928. doi:10.1016/j.comcom.2012.02.004

URL http://www.sciencedirect.com/science/ article/pii/S0140366412000515

[157] Z. Qu, D. Chen, G. Sun, X. Wang, X. Tian, J. Liu, Efficient wireless sensor networks scheduling scheme: Game theoretic analysis and algorithm, in: Communications (ICC), 2012 IEEE International Conference on, IEEE, 2012, pp. 356-360.

[158] H. Liu, G. Liu, Y. Liu, L. Mo, H. Chen, Adaptive quantization for distributed estimation in energy-harvesting wireless sensor networks: A game-theoretic approach, International Journal of Distributed Sensor Networks 2014. 